\title{
Die „Gesundung“ der Altstädte und der Beginn der städtebaulichen Denkmalpflege um 1900
}

Um die Assanierung der Stadt Wien in den Jahren des Ständestaats analysieren und bewerten zu können, muss man die Ereignisse in einem breiten stadtgeschichtlichen und architekturhistorischen Kontext betrachten, denn mit den Maßnahmen der „Stadtgesundung“ wurde in den 1930er-Jahren eine bereits vor dem Ersten Weltkrieg begonnene Entwicklung fortgesetzt: die sukzessive Erneuerung und Regulierung historischer Stadtbereiche. Zu Beginn dieses einführenden Kapitels sollen daher die Regulierungs- und „Gesundungsmaßnahmen“ der letzten Jahrzehnte der Monarchie und die dadurch entstandenen Veränderungen des Stadtbildes - in der notwendigen Kürze - besprochen werden. Durch diesen etwas geweiteten Fokus werden Kontinuitäten in der Stadtplanung von der Jahrhundertwende bis in die späten 1930er-Jahre und darüber hinaus deutlich.

Der Blick auf die Geschichte der Denkmalpflege und des Denkmalschutzes in Österreich zu Beginn des 20. Jahrhunderts wird weitere wesentliche Anhaltspunkte für die Bewertung der Ereignisse der 1930er-Jahre liefern. Die Denkmalpflege als wissenschaftliche Disziplin begann sich in den Jahren nach 1900 mit städtebaulichen Fragen zu befassen und widmete sich zunehmend dem „Problem Altstadt“. Man suchte gleichsam nach geeigneten Methoden für die Erhaltung wie für die „Gesundung“ der dicht verbauten historisch „gewachsenen“ Stadtkerne. Dieser Diskurs wird in der Zwischenkriegszeit wiederaufgenommen und intensiviert. Der Blick zurück auf die Ereignisse um 1900 in den Bereichen Städtebau und Denkmalpflege ist unumgänglich und aufschlussreich, um historische Zusammenhänge zu erkennen und längere Entwicklungen zu veranschaulichen.

\subsection{Wandel des Wiener Stadtbildes ab dem 19. Jahrhundert}

Den „Gesundungsmaßnahmen“ der 1930er-Jahre gingen wesentliche, das Stadtbild und die Stadtstruktur prägende Eingriffe im Verlauf des 19. Jahrhunderts voraus. Wie in vielen anderen europäischen Städten wurde auch in Wien um die Mitte des 19. Jahrhunderts nicht nur ein Stadterweiterungs-, sondern auch ein gewaltiger Stadtumbauprozess - aus wirtschaftlichen, verkehrstechnischen und auch aus „hygienischen“ Gründen - in Gang gesetzt.' Die beiden ersten Gründe müssen hier nicht näher erläutert werden, hingegen umso mehr der Begriff der Hygiene.

Die europäische Stadt war ab dem 19. Jahrhundert unterschiedlichen Maßnahmen der „Gesundung“, also der Assanierung, unterworfen. Der Begriff Assanierung etablierte sich als Bezeichnung für eine „sanitäre Stadtregulierung“ im Laufe des 19. Jahrhunderts, nachdem der Hygiene-Diskurs in Frankreich bereits in der Aufklärung eingesetzt und sich im 19. Jahrhundert auf die Dimensionen der Stadt und die Interessen des Städtebaus ausgeweitet hatte. ${ }^{2}$ Unter der „Gesundung“ verstand man städtebauliche Maßnahmen wie die Regulierung und Auflockerung des Altstadtgefüges durch Straßenverbreiterungen oder Straßendurchbrüche. Dadurch sollte die Zufuhr von 
ausreichend Licht und Luft in die historisch gewachsenen und dicht bebauten Stadtbereiche ermöglicht und damit bessere Lebensbedingungen geschaffen werden. Ebenso verstand man darunter den Ausbau der Kanalisation, eine ausreichende Wasserversorgung oder die Anlage öffentlicher Gärten.

Der Umbau oder gar Abbruch der historischen - vor allem der mittelalterlich geprägten - Stadtbereiche und die Verbesserung der dort herrschenden Wohnverhältnisse wurde von den Vertretern der Stadterneuerung als zwingend erforderlich erachtet. Die hygienischen Aspekte wurden in diesem Diskurs, so Renate Banik-Schweitzer, auch mit wirtschaftlichen Überlegungen verknüpft. „Ungesund“ und „unhygienisch“ wurde mit „wenig ertragreich" nahezu gleichgesetzt und die betroffenen Stadtquartiere dadurch stigmatisiert. ${ }^{\mathbf{3}}$ Aufgrund der niedrigen Mieteinnahmen waren die Besitzer dieser Häuser an Instandsetzungsmaßnahmen zumeist nicht interessiert. In den baulich schlecht erhaltenen Altstadtbereichen siedelten sich vor allem einkommensschwache Schichten an und die Altstadt wurde zunehmend auch mit sozialen Missständen in Verbindung gebracht. 4

Diverse Interessen führten also vor allem in der zweiten Hälfte des 19. Jahrhunderts zu weitgreifenden Veränderungen und einem massiven Stadtumbau in Wien und in zahlreichen anderen europäischen Großstädten. ${ }^{\mathbf{5}}$ Zwar setzte eine entscheidende bauliche Umgestaltung der Stadt schon im späten 18. Jahrhundert mit der Liberalisierung des Bauwesens unter Josef II. ein, ${ }^{\mathbf{6}}$ doch erst mit den städtebaulichen Eingriffen der Gründerzeit erreichte diese Entwicklung ihren Höhepunkt.

Der wirtschaftliche Aufschwung und die damit einhergehende Citybildung ${ }^{\mathbf{7}}$ führten ab etwa 1840 zum Umbau des Stadtzentrums zu einer Geschäftsstadt, was sich in der Architektur und im Stadtgrundriss bemerkbar machte. Wie Banik-Schweitzer bereits darlegte, geschah dies in Wien hauptsächlich durch einen parzellenweisen Umbau, also durch eine schrittweise Regulierung. ${ }^{\mathbf{8}}$ Ausgeführt wurde diese durch private Hausbesitzer oder große Baugesellschaften und vor allem entlang der Hauptverkehrsstraßen. In den dahinterliegenden, verkehrsarmen Bereichen blieb die alte Bausubstanz hingegen zumeist erhalten. ${ }^{9}$ Nicht zuletzt, da sich die Gemeinde aus finanziellen Gründen keine umfangreichen Enteignungen leisten konnte, wurden nur einige wenige Straßenverläufe planmäßig reguliert, wie die Verbreiterung der Einmündung des Grabens in den Stephansplatz im Jahr 1866. Durch den parzellenweisen Umbau nahmen Straßenverbreiterungen zumeist viele Jahre bis Jahrzehnte in Anspruch, wie im Falle der Regulierung der Kärntner Straße und des Neuen Marktes. ${ }^{10}$ Dennoch handelte es sich bei den Regulierungsmaßnahmen der Gründerzeit - im Gegensatz zu anderen wirtschaftlich, politisch und gesellschaftlich motivierten Umbauphasen in der Geschichte der Stadt - um weitreichende Eingriffe, die eine rasante Veränderung von Stadtstruktur und Stadtbild zur Folge hatten. Die Generalstadtpläne des frühen 20. Jahrhunderts oder Baualterpläne der Inneren Stadt verdeutlichen dies.

Dieser „privatwirtschaftliche Städtebau“ vor 1914 hatte nicht nur auf die bauliche, sondern auch auf die soziale Struktur der Innenstadt entscheidende Auswirkungen - ein, laut Harald Bodenschatz, allgemeines Phänomen: Dort, wo sich diese Umwandlung der Altstadt nämlich nicht vollzog, weil die Gebäudestruktur oder die Lage sich nicht dafür anboten, blieben zwar die malerischen Ensembles erhalten, jedoch verkamen die historischen Stadtbereiche dadurch häufig zu Wohnbereichen der ärmeren Schichten. ${ }^{11}$ Es entstand ein Nebeneinander von Geschäftsstadt und „Elendsvierteln“. 
1936 beklagte die Zentralstelle in der Zeitschrift Deutsche Kunst und Denkmalpflege die weitreichende Zerstörung der historischen Stadtbereiche Wiens in der damals jüngsten Vergangenheit, vor allem in den Jahren zwischen 1890 und $1914 .{ }^{\mathbf{1 2}}$ Adalbert Klaar, ${ }^{\mathbf{1 3}}$ ab 1946 Mitarbeiter des Bundesdenkmalamtes, bezeichnete den ab den 1850er-Jahren einsetzenden Stadtumbau, der vor allem in der Kernstadt und in den angrenzenden Bezirken durch die Anlage von Durchbruchstraßen und die Verbreiterung und Begradigung alter Verkehrswege großräumige Altstadtgefüge zerstört habe, rückblickend als „zersetzenden und verdichtenden Städtebau“. In den wenigen Jahren zwischen 1875 und 1918 wurden seinen Angaben nach mehr Altbauten demoliert als in den Jahrhunderten davor. ${ }^{14}$

Das Stadtbauamt war hier freilich anderer Ansicht. Nach Auffassung von Heinrich Goldemund, ab 1913 Stadtbaudirektor, war die Regulierung und Assanierung Wiens im 19. Jahrhundert zu wenig konsequent und zu langsam durchgeführt worden. In einem Zeitraum von lediglich zehn Jahren (1859-1869) seien allerdings im Zuge der Stadterweiterung 1.525 Häuser neu errichtet und 2.836 Althäuser umgebaut worden, was sich „sehr günstig“ auf die sanitären Verhältnisse ausgewirkt habe. ${ }^{15} 1923$ relativierte er jedoch seine Position und forderte eine Überprüfung des bestehenden Regulierungsplanes. ${ }^{16}$

Vor allem ab der Mitte des 19. Jahrhunderts veranlassten der Gemeinderat und neu geschaffene Einrichtungen, wie das Stadtbauamt (gegründet 1835) und die Donauregulierungskommission (seit 1867), städtebauliche Maßnahmen, die weitreichende Veränderungen von Stadtbild und Stadtstruktur nach sich zogen. Der Abriss der Befestigungsanlagen um den heutigen 1. Bezirk sowie der Abbruch des Linienwalls beseitigten auf radikale Weise historische Zeitschichten der Stadt. Die dadurch ermöglichte Stadterweiterung und bauliche Erschließung des Glacis vollzog sich in unglaublichem Tempo. Der Ausbau der Kanalisation, die Regulierung der Donau, des Donaukanals und des Wienflusses, der Bau der Stadtbahn und zahlreicher städtischer Versorgungseinrichtungen brachten jedoch auch soziale und hygienische Verbesserungen mit sich. Mit der Einführung der ersten Wiener Bauordnung 1829 wurde andererseits aber auch ein erstes Regelwerk geschaffen, um die ausufernde private Bautätigkeit in geordnete Bahnen zu lenken. ${ }^{\mathbf{7}}$

Nach der Eingemeindung der Vororte in den Jahren 1850 und 1892 wurden auch für diese Stadtbereiche Regulierungspläne erstellt, nachdem sich die erste bauliche Verdichtung und Erweiterung meist relativ planlos vollzogen hatten. ${ }^{\mathbf{1 8}}$ Max Dvořáks Kritik im Vorwort zum 2. Band der Österreichischen Kunsttopographie von 1908 nimmt einige Jahre später auf ebendiese - und fortwährende - Entwicklung Bezug und ortet gerade hier beunruhigende Veränderungen:

„Am schnellsten vollzieht sich [...] dieser Prozeß in den an der Peripherie liegenden Stadtvierteln, wo wohl kaum ein Tag ohne gewaltsame Eingriffe in das alte Stadtbild vergehen dürfte, so daß nicht etwa in einigen Dezennien, sondern in einigen Jahren von den einstigen anmutigen Vorstädten kaum mehr übrig bleiben dürfte als eine literarische Reminiszenz in alten Beschreibungen und den Liedern der Volkssänger.“19 
Die Wiener Bauordnung wirkte sich seit ihrer Einführung 1829 durch ihre Vorschriften zu städtebaulichen Fragen und zur architektonischen Gestaltung entscheidend auf das Wiener Stadtbild aus. ${ }^{\mathbf{2 0}}$ Vor allem die Regelungen zu Gebäudehöhen und dazu entsprechenden Mindeststraßenbreiten prägten fortan die Entwicklung der Stadt. Die von der alten Baulinie zurückrückenden Neubauten zerpflückten die einst geschlossenen Baulinien und Straßenzüge und erzwangen letztlich geradezu den Abriss der daran angrenzenden Altbauten.

Mit der Bauordnungsnovelle von 1890 wurde schließlich die Ausarbeitung eines das gesamte Gemeindegebiet umfassenden Generalregulierungsplans gesetzlich verankert. ${ }^{21}$ Im Jahr 1892 fand ein internationaler Wettbewerb statt, der dafür Ideen und Grundlagen beschaffen sollte. ${ }^{22}$ Eine zentrale Vorgabe war dabei, leistungsfähige Verkehrsadern einzuplanen und die Struktur der (Alt-)Stadt im Hinblick auf eine verkehrstechnische Erschließung zu „verbessern“, aber auch auf die künstlerische Ausgestaltung Rücksicht zu nehmen. Die beiden ersten Plätze gingen an die Konzepte von Otto Wagner und Joseph Stübben. ${ }^{\mathbf{2 3}}$ Zur Ausarbeitung eines endgültigen Generalregulierungsplanes wurde im Jahr 1894 eine eigene Bauamtsabteilung gegründet und Heinrich Goldemund und Karl Mayreder als Leiter berufen. ${ }^{\mathbf{2 4}}$ Aufgrund des Umfangs der Arbeit und des enormen Bauaufkommens kam es jedoch nie zur Erstellung eines zusammenhängenden Planwerks für das gesamte Stadtgebiet, der Generalregulierungsplan blieb ein Konvolut einzelner Teilregulierungspläne. ${ }^{25}$ In den Regulierungsplänen dieser Jahre wurden, basierend auf den Vorschriften der Bauordnung, zahlreiche neu gezogene Baulinien festgelegt, die in den Jahrzehnten danach mit der Errichtung der Neubauten auch realisiert wurden. Ohne Rücksicht auf die gewachse-

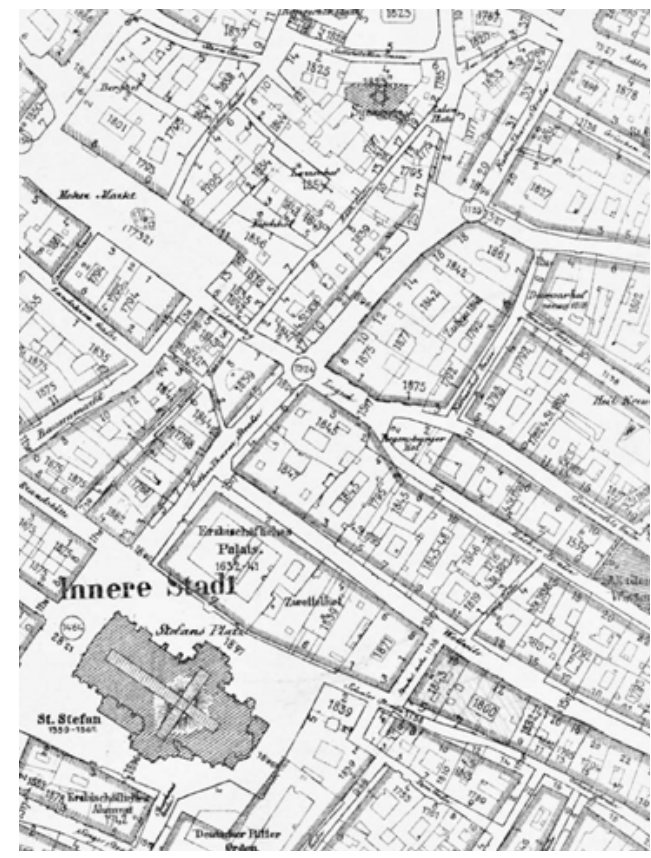

Abb. 4 Generalregulierungsplan, Ausschnitt Rotenturmstraße, schraffiert dargestellt die genehmigten neuen Baulinien, 1892 nen Strukturen der Stadt versuchte man, dem verwinkelten Stadtgefüge ein geradliniges und damit „verkehrstaugliches“ Straßenraster aufzuzwingen (Abb.4).

Das Stadtbauamt konnte seine Regulierungsabsichten nahezu ungehindert durchsetzen, denn Regulierungspläne mussten $a b$ der Bauordnungsnovelle von 1890 nach Vorlage im Stadtrat nur noch vom Gemeinderat abgesegnet werden, und in den Gemeinderatssitzungen gab es kaum Diskussionen um einzelne Regulierungsvorhaben. ${ }^{\mathbf{2 6}}$ Einige radikale Pläne blieben jedoch auf dem Papier. So plante etwa Karl Mayreder 1895 drei die Verkehrssituation entlastende neue Straßenzüge durch die Innere Stadt (Abb. 5) - Pläne, die vor allem von Camillo Sitte stark kritisiert wurden. ${ }^{27}$ Mayreders Entwurf fußte, laut eigener Aussage, auf der Überlegung, „der Inneren Stadt bei der nothwendigen Regulirung ihre eigenartige Individualität möglichst zu erhalten“28. Dabei plante er allerdings auch einen Straßendurchbruch vom Laurenzerberg über 


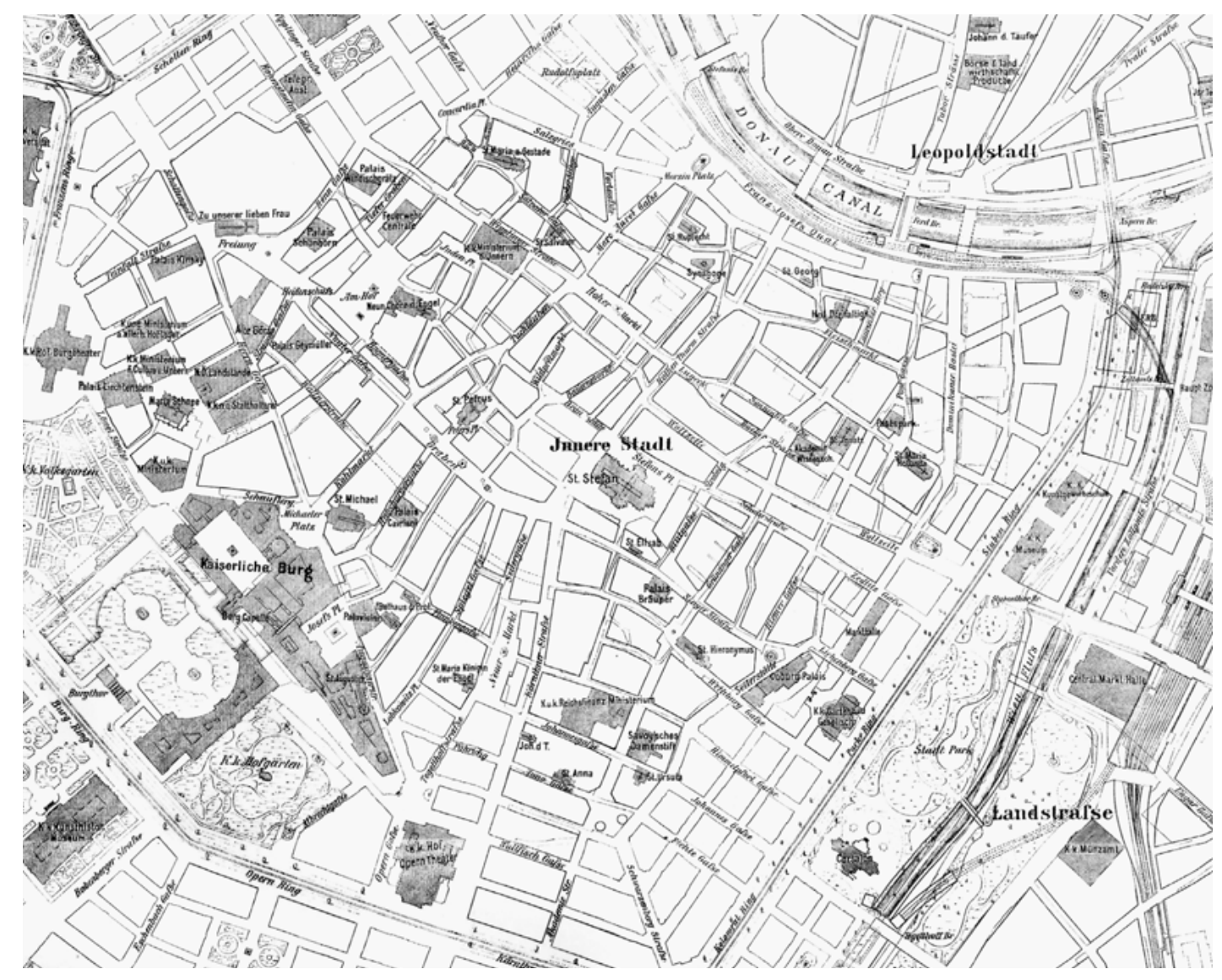

Abb. 5 Karl Mayreder, Regulierungsplan Innere Stadt, 1895

den Stephansplatz bis zur Akademiestraße, der eine Schneise durch die gesamte Innenstadt geschlagen und unter anderem die alten Stadtbereiche an der Johannes- und Himmelpfortgasse sowie eine Platzwand des Franziskanerplatzes durchbrochen hätte. ${ }^{29}$ Der Straßendurchbruch wurde auch bewilligt, letztlich aus finanziellen Gründen aber nicht durchgeführt (Abb. 6). ${ }^{\mathbf{3 0}}$ Weitere Utopie gebliebene Themen waren bis zum Ausbruch des Ersten Weltkrieges auch die Führung einer Straßenbahn durch die Altstadt und unterirdische Durchquerungen des Stadtkerns mit Untergrundbahnen. ${ }^{31}$

In den Jahren nach 1900 bis zum Ersten Weltkrieg wurde die intensive Demolierungstätigkeit fortgeführt. Als unmittelbare Reaktion der institutionellen Denkmalpflege mangelt es im 15. Band der Österreichischen Kunsttopographie von 1916 zum erhaltenswerten Baubestand der gesamten Reichshaupt- und Residenzstadt Wien nicht an Kritik an der Stadtentwicklung der Gründerzeit und der Jahrhundertwende. ${ }^{\mathbf{3 2}}$ Wichtige Ausfallstraßen, wie die Lerchenfelder Straße, die Landstraßer Hauptstraße oder die Gumpendorfer Straße, erfuhren in dieser Zeit eine umfangreiche Regulierung und „Modernisierung“. ${ }^{33}$ Auch in der Zwischenkriegszeit bis 1934 wurden zahlreiche Gebäude abgetragen und dadurch die Regulierung und „Harmonisierung“ des Stadtgrundrisses durch möglichst geradlinig verlaufende Baulinien weiter vorangetrieben. ${ }^{\mathbf{3 4}}$ In 
den Jahren 1925 bis 1934 demolierte man allein in der Sieveringer Straße (19. Bezirk) neun Häuser, im Jahr 1936 weitere fünf Objekte. ${ }^{35}$ Grundsätzlich ist das Ausmaß der Regulierungstätigkeit dieser Jahre aber nicht mit jenem vor dem Ersten Weltkrieg und nach 1934 vergleichbar. Die städtebauliche Hauptaufgabe der sozialistischen Stadtregierung konzentrierte sich ab den frühen 1920er-Jahren auf den Bau der großen Wohnhausanlagen, die zumeist an der damaligen Peripherie errichtet wurden. Das kommunale Wohnbauprogramm belastete die finanziellen Ressourcen dermaßen, dass die Stadtverwaltung an städtebaulichen Planungen und der „Gesundung“ der Altstadt wenig Interesse zeigte. ${ }^{\mathbf{3 6}}$ Darüber hinaus verfügte man über keinerlei fortschrittliche Planungsmethoden. ${ }^{37}$

Neben den Regulierungsplänen und Bauvorschriften in Bauordnungen begünstigten auch andere gesetzliche Vorgaben ab dem späten 19. Jahrhundert die rasch fortschreitende Regulierung: Bereits im Jahr 1880 hatte man ein Gesetz verabschiedet, das beim Um- bzw. Neubau des Eigenheimes eine Steuerbefreiung für die Dauer von zwölf Jahren zugestand. Das Gesetz sollte Hausbesitzer zum Umbau ihrer Liegenschaften motivieren und dadurch auch die Beseitigung verkehrsbehindernder Straßenengen begünstigen. ${ }^{38}$ Im Jahr 1893 wurde die Dauer der Steuerbefreiung auf 18 Jahre ausgedehnt und mit dem Zusatz ,anlässlich von Regulierungen und Erweiterungen der Hauptverkehrsstraßen“ die Zweckbestimmung noch genauer definiert. ${ }^{39}$ Allein in der

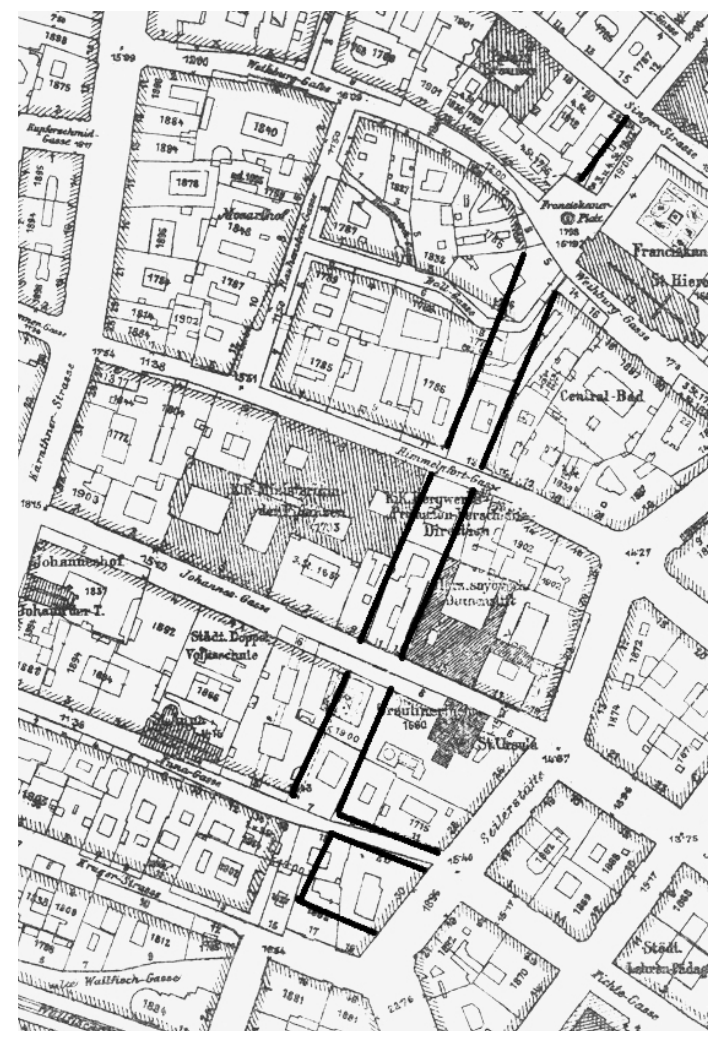

Abb. 6 Generalstadtplan, projektierter Straßendurchbruch vom Franziskanerplatz bis zur Krugerstraße (hier hervorgehoben), 1912
Inneren Stadt mussten dieser Offensive bis in die ersten Jahre des 20. Jahrhunderts etwa 766 Häuser weichen. ${ }^{40}$

Eine entscheidende Änderung erfolgte mit der neuen Bauordnung für Wien von 1929, die die gesetzlichen Bestimmungen zur Enteignung enthielt und damit die Möglichkeiten der Stadt Wien in der Umsetzung städtebaulicher Planungen bedeutend erweiterte. ${ }^{\mathbf{4 1}}$ Somit war es fortan möglich, „wenn mangelnder Verkaufswille oder übertriebene Forderungen die im Interesse der baulichen Entwicklung der Stadt wichtigen Projekte zu verhindern [drohten]“, bebaute und unbebaute Grundstücke oder Grundstücksteile gegen Entschädigung zu erwerben oder per Enteignungsverfahren den Verkauf zu erzwingen. Selbiges galt laut Bundesgesetz explizit auch für „Baulichkeiten, die infolge ihres gesundheitswidrigen oder baufälligen Zustandes abbruchreif [waren] oder deren Umbau aus öffentlichen Rücksichten notwendig [erschien]“.42 In Städtebaukongressen hatte man diese Ausweitung des rechtlichen Rahmens schon länger vehement gefordert und in Deutschland, 
den ehemaligen Kronländern und in anderen Bundesländern war das Enteignungsrecht in den Bauordnungen bereits vor 1900 verankert worden. ${ }^{\mathbf{4 3}} \mathrm{Ab} 1930$ galt dieses nun auch für Wien, bei der Anlage von neuen oder der Verbreiterung von bestehenden Verkehrsflächen, für Baulücken, Ergänzungsflächen, zur Anlage von Friedhöfen und öffentlichen Erholungsflächen und zu Assanierungszwecken. Aufgrund der finanziellen Lage der Gemeinde wurden Enteignungen jedoch kaum durchgesetzt (siehe dazu später noch Kap. 6.4.2.).

Einen ersten Anreiz für die private Bautätigkeit - in einer wirtschaftlich äußerst angespannten Situation - gab es bereits vor Einführung des Assanierungsfonds mit dem 1929 von der christlich-sozialen Bundesregierung verabschiedeten Wohnbauförderungsgesetz und der Einführung der Befreiung von der Wohnbausteuer in Wien im selben Jahr. ${ }^{44}$ Vor allem für die Jahre 1929 und 1930 ist auch in den Akten im Archiv des Bundesdenkmalamtes ein merklicher Anstieg an Abbruchsansuchen zu verzeichnen, der sicherlich auf diese gesetzlichen Änderungen zurückzuführen ist.

Zusammenfassend lässt sich festhalten, dass in Wien ab der Mitte des 19. Jahrhunderts eine verstärkte Abrisstätigkeit stattfand, die sich entscheidend auf die Stadtstruktur und das Stadtbild auswirkte und in den Jahren des Ständestaats einen neuerlichen Höhepunkt erreichte. In der Gründerzeit erfolgte die wohl intensivste Umbauphase Wiens mit der Anlage der Ringstraße, dem Umbau der Inneren Stadt zu einer Geschäftsstadt und der Stadterweiterung. Begünstigt wurde diese Entwicklung durch den wirtschaftlichen Aufschwung, die neu geschaffenen gesetzlichen Rahmenbedingungen, soziale Veränderungen und die Notwendigkeit, die räumliche Ausdehnung der Stadt zu ermöglichen. Zahlreiche Demolierungen und Regulierungsmaßnahmen der 1930er-Jahre wurden bereits Jahrzehnte zuvor angedacht und in den Regulierungsplänen festgehalten. ${ }^{45}$ Betroffen waren vor allem Straßenzüge mit unregelmäßigem Verlauf oder uneinheitlichen Baulinien. Anhand des Regulierungsplans und dank der vorrangig privaten Bautätigkeit sollten zahlreiche Straßenverläufe der Inneren Stadt, aber auch der umliegenden Bezirke, in ein möglichst rasterartiges, geradliniges Straßensystem umgewandelt werden. Wie einige Beispiele zeigen werden, griff man noch in den Jahren des Ständestaats auf eine zu diesem Zeitpunkt mittlerweile rund 40 Jahre alte - und in den Augen zahlreicher Fachleute und Architekten veraltete - stadtplanerische Grundlage zurück. Die im Laufe des 19. Jahrhunderts erfolgte parzellenweise Regulierung führte man in den Jahren des Ständestaats weiter fort. Eine große Zahl Althäuser, vorrangig aus dem 18. und frühen 19. Jahrhundert, die seit dem Stadtumbau der Gründerzeit und den damals geänderten Baubestimmungen oftmals weit in den Straßenraum vorstanden, wurden im Zuge dessen aus dem Stadtbild entfernt.

Somit zeigt sich eine lineare Entwicklung und konsequente Umsetzung städtebaulicher Regulierungsmaßnahmen vom späten 19. Jahrhundert bis zum Ende der 1930er-Jahre. Neben der Einführung des Assanierungsfonds beschleunigten auch entscheidende gesetzliche Änderungen wie die Steuerbefreiungen bei Um- und Neubauten (1880) ebenso wie die erweiterten Enteignungsbestimmungen (1929) die fortschreitende Regulierung der Stadt. 


\subsection{Stadterhaltung und Denkmalpflege}

Einhergehend mit der Entwicklung der Stadtplanung und den Regulierungsmaßnahmen des 19. Jahrhunderts formierten sich im deutschsprachigen Raum die Institution und wissenschaftliche Disziplin der Denkmalpflege. ${ }^{\mathbf{4 6}}$ In die Phase der umfangreichen Zerstörung historischer Bausubstanz und Erneuerung zahlreicher Städte fiel auch die Gründung der „k.k.Central-Commission für die Erforschung und Erhaltung der Baudenkmale" im Jahr 1850.47

Nicht zuletzt als eine Folge des Stadtumbaus der Gründerzeit wird um 1900 und vor allem im frühen 20. Jahrhundert ein wachsendes Interesse an der Orts- und Stadtbildpflege, eine steigende Wertschätzung des Ensembles und ein zunehmendes Bewusstsein für die Bedeutung der Umgebung von Denkmalen deutlich. ${ }^{\mathbf{4 8}}$ Vor allem die im 19. Jahrhundert übliche Freistellung von Monumentalbauten durch den Abbruch umgebender Bauwerke wird heute als einer der Auslöser für die Entwicklung städtebaulich-denkmalpflegerischer Überlegungen gesehen. ${ }^{49}$ Alois Riegls Untersuchung der Altstadt von Split und des geplanten Abbruchs zahlreicher Gebäude um die Kathedrale (das ehemalige Diokletian-Mausoleum) im Jahr $1903^{\mathbf{5 0}}$ ist ein Hinweis darauf, dass bereits kurz nach der Jahrhundertwende die Wertschätzung für Ensembles und gewachsene bauliche Zusammenhänge in der österreichischen Denkmalpflege angekommen war. Riegls abschließendes Gutachten sprach sich entschieden für die Erhaltung der gewachsenen Stadtstruktur und des malerischen Stadtbildes aus und gegen eine Freilegung der Kathedrale und der Überreste des Diokletianspalastes.

Somit nahmen auch in der Denkmalpflege Diskurse zum Thema der (Alt-)Stadterhaltung zunehmend Raum ein, nachdem Termini wie „Stadtbild“ und „Straßenbild“ bis zur Jahrhundertwende zum Vokabular des Städtebaus gezählt hatten und die Stadtbildpflege zu den Aufgaben der Bauämter.51 Auch der Begriff „Altstadt“ selbst etablierte sich nach der Jahrhundertwende, vor allem als Abgrenzung zu den jüngst entstandenen Gründerzeitvierteln. ${ }^{52}$

Für den „Umbau alter geschichtlicher Städte unter Berücksichtigung neuzeitlicher Forderungen"53 endlich Lösungen zu finden, gewann für beide Disziplinen nach und nach an Bedeutung: Der ab 1900 jährlich stattfindende „Tag für Denkmalpflege“54 wurde gleichsam zum wichtigsten Ort für die Diskussion von Aspekten der Altstadt- und Stadt(bild)erhaltung. ${ }^{\mathbf{5 5}}$ Einhergehend mit der Kritik an einem rücksichtslosen Umgang mit der „Alten Stadt“ wurden nun seitens der Denkmalpflege auch erste konkrete städtebauliche Lösungen präsentiert. Im Rahmen der Tagung in Erfurt im Jahr 1903 referierten Joseph Stübben und Cornelius Gurlitt - ihres Zeichens Architekten und Fachleute des Städtebaus - über „die Gestaltung der Straßenfluchtlinien vom Standpunkte der Denkmalpflege“ und brachten ihre Anschauungen in „trefflich formulierten Leitsätzen“56 zu Papier. Zwei Jahre später in Bamberg betonte Paul Jonas Meier die Bedeutung historischer Stadtstrukturen mit ihren Straßenverläufen und Platzräumen und bezeichnete den Grundriss der Stadt als „die monumentalste Urkunde ihrer Geschichte“57. 1907 sprach man in Mannheim „Über die Möglichkeit der Erhaltung alter Stadtbilder unter Berücksichtigung moderner Verkehrsanforderungen"58 und im Rahmen der ersten Gemeinsamen Tagung von Denkmalpflege und Heimatschutz in Salzburg im Jahr 1911 referierte Cornelius Gurlitt über die „Erhaltung des Kernes alter Städte“.59 1916 äußerte sich schließlich die staatliche Denkmalpflege in der Österreichischen Kunsttopographie sehr kritisch zu den Folgen der Citybildung in der Inneren Stadt. ${ }^{60}$ 
Nach einigen Jahren der Unterbrechung durch den Ersten Weltkrieg wurde die Diskussion der Altstadtfragen in den Reihen der Denkmalpflege Ende der 1920er-Jahre fortgesetzt und intensiviert. 1928 befasste man sich am Tag für Denkmalpflege und Heimatschutz in Würzburg und Nürnberg ausführlich mit dem Thema „Altstadt und Neuzeit", mit Grundsatzfragen, Verkehrsproblemen bis hin zur Farbe im Stadtbild. ${ }^{61}$ Diese Tagung und das Verhältnis der Denkmalpflege zur Stadt in den 1930er-Jahren wird später noch von Interesse sein.

Aber nicht nur die Denkmalpflege, sondern auch Vertreter des Städtebaus beschäftigten sich um 1900 mit der Frage der Erhaltung und des Umgangs mit der historischen Stadt. Erstmals in großer Form geschah dies im Rahmen der Allgemeinen StädtebauAusstellung in Berlin 1910. Die Resonanz war enorm, sodass Teile der Ausstellung auch in London gezeigt wurden. ${ }^{62}$ Unter den Exponaten waren aktuelle und weiter zurückliegende durchgeführte und geplante Regulierungsmaßnahmen in diversen europäischen und außereuropäischen Städten, darunter auch zahlreiche Beispiele aus Wien. ${ }^{\mathbf{6 3}}$ Man zeigte Teilregulierungspläne zu einzelnen Stadtbereichen, Studien zur Regulierung des Karlsplatzes sowie den geplanten Straßendurchbruch parallel zur Kärntner Straße, der mit der Absicht geplant sei, „den schönen alten Monumentalbauten tunlichst aus dem Wege zu gehen und bestehende alte malerische Stadtbilder nicht $\mathrm{zu}$ stören“.64 International - wie auch national - stieß der Durchschlag durch die Wiener Altstadt, der letztlich nicht realisiert wurde, dennoch auf harte Kritik. ${ }^{\mathbf{6 5}}$ Es folgten erste deutschsprachige Fachpublikationen, die sich mit dem Thema Stadtsanierung und Stadtregulierung auseinandersetzten, wie Gurlitts Handbuch des Städtebaues und Otto Schillings Innere Stadterweiterung. ${ }^{\mathbf{6}}$

Diese steigende Wertschätzung des Stadtbildes und der Altstadt im Allgemeinen lässt sich auf die vorausgegangene Bewusstseinsbildung um die Bedeutung ästhetischer Aspekte im Städtebau zurückführen. ${ }^{\mathbf{6 7}}$ Nach der stark technischen Ausrichtung des Städtebaus im 19. Jahrhundert hatte erstmals Camillo Sitte mit Der Städte-Bau nach seinen künstlerischen Grundsätzen (1889) die Bedeutung ästhetischer Fragen im Städtebau festgehalten. Um 1900 erschienen in weiterer Folge zahlreiche Publikationen, wie Karl Henricis Die künstlerischen Aufgaben im Städtebau (1891), Charles Buls L'esthéthique des villes (1893) oder Albert Erich Brinckmanns Platz und Monument. Untersuchungen zur Geschichte und Ästhetik der Stadtbaukunst in neuerer Zeit (1908). ${ }^{68}$ Die Wertschätzung der Altstadt wurde mit dieser Entwicklung gleichsam mitgetragen.

Einhergehend mit dem wachsenden Bewusstsein für die historische und künstlerische Bedeutung der Altstädte etablierte sich um 1900 neben dem städtebaulichen auch ein denkmalpflegerisches Interesse an der Erhaltung der historischen Stadt, das der ästhetischen Wirkung des Stadtbildes und der Erhaltung des Ensembles eine wachsende Bedeutung zuschrieb und sich gegen das rein technokratische, räumlich-strukturelle Verständnis der Stadt und die damit einhergehende verkehrstechnische und „hygienische“ Regulierung auflehnte. ${ }^{69}$

Die im frühen 20. Jahrhundert festzustellende Erweiterung des ursprünglichen Denkmalbegriffs und die Ausdehnung des Aufgabenspektrums der Denkmalpflege auf den Ortsbildschutz lässt sich aber auch auf den Einfluss der Heimatschutzbewegung zurückführen, die in Deutschland bereits 1904 gegründet wurde. ${ }^{\mathbf{7 0}}$ In Österreich formierte sich die Heimatschutzbewegung 1908 in Tirol und bestand vorerst aus einzelnen 
regionalen Gruppierungen, die sich 1912 im „Verband Österreichischer Heimatschutzvereine“ zusammenschlossen. ${ }^{\mathbf{1 1}}$ Die „Volksbewegung“72 des Heimatschutzes befasste sich mit der Bewahrung des kulturellen Reichtums, der Volkskunde und der Baukultur. Im Gegensatz zur institutionalisierten Denkmalpflege, der wissenschaftlichen Disziplin zur Erforschung und Erhaltung der Denkmale, sah man sich als „volksnahe Bewegung" und widmete sich nicht nur denkmalpflegerischen Anliegen im Allgemeinen, sondern vor allem auch dem Ortsbildschutz. ${ }^{\mathbf{7 3}}$ Die Erhaltung der Wirkung des Denkmals in seiner Umgebung und den Schutz größerer Stadtzusammenhänge und Ensembles verstand man als eine der zentralen Aufgaben. ${ }^{\mathbf{7 4}}$ Das „künstlerisch schöpferische Moment“ spielte dabei allerdings schon seit den Anfängen eine gewichtige Rolle, was Karl Giannoni ${ }^{\mathbf{7 5}}$, der führende Kopf der österreichischen Heimatschutzbewegung, im Rahmen der Gemeinsamen Tagung für Denkmalpflege und Heimatschutz in Salzburg 1911 als den eigentlich einzigen „wirklich unterscheidende[n] Faktor auf dem der Denkmalpflege und dem Heimatschutz gemeinsamen Gebiete der Erhaltung des Ortsbildes“ bezeichnete. ${ }^{\mathbf{7 6}}$ Man unterstützte keine „museale“ Konservierung der Stadt, sondern verstand sich als Förderer der heimischen Baukultur und befürwortete auch im Bereich der Altstadt die Errichtung „angemessener“ zeitgenössischer Bauten. Unter den über 800 Anwesenden waren laut Teilnahmeliste aus Österreich zahlreiche Mitarbeiter der institutionellen Denkmalpflege, ${ }^{\mathbf{7 7}}$ aber interessanterweise auch einstige Mitgestalter der Wiener Stadtregulierung, Oberbaurat Heinrich Goldemund und Karl Mayreder.

Nicht zuletzt verstärkte die umfangreiche Bau- und Regulierungstätigkeit der Gründerzeit ab der Mitte des 19. Jahrhunderts die Forderungen nach gesetzlichen Schutzmaßnahmen für den gefährdeten Baubestand. Konkrete Beratungen diesbezüglich setzten schließlich in den $1890 e r-J a h r e n$ ein. 1898 brachte Joseph Alexander Freiherr von Helfert schließlich einen ersten Gesetzesentwurf im Herrenhaus vor, gefolgt von einem neuerlichen Entwurf 1903 mit dem bekannten Vorwort Alois Riegls zu „Wesen und Entstehung des modernen Denkmalkultus““78 Auf die Notwendigkeit einer Gesetzesgrundlage verweist auch Karl Holey 1911: „Und wann wäre ein starker Schutz nötiger als heute. Es hat gewiß nicht viele Zeiten gegeben, in denen die Zerstörung der alten Denkmäler gründlicher und grundloser betrieben worden wäre als in den letzten Jahren des XIX. Jahrhunderts."79

Bis zur Verabschiedung des österreichischen Denkmalschutzgesetzes ${ }^{\mathbf{8 0}}$ dauerte es allerdings noch bis zum Jahr 1923. Dem umfassenden Schutz des beweglichen und unbeweglichen Denkmalbestandes kam die Denkmalschutzbehörde damit einen wesentlichen Schritt näher, die unmittelbaren Auswirkungen waren vorerst jedoch minimal, auch aufgrund der schlechten personellen und finanziellen Situation. ${ }^{\mathbf{8 1}}$ Darüber hinaus war der Schutz von Ensembles und städtischen Bereichen durch das Denkmalschutzgesetz weiterhin nicht gegeben. Der Orts- oder Stadtbildschutz sollte erst ab den 1970er-Jahren österreichweit eingeführt werden, gesetzlich verankert in den jeweiligen Landesgesetzen - in den Bauordnungen und Raumordnungsgesetzen. ${ }^{\mathbf{8 2}} 1978$ wurde schließlich das Ensemble in das österreichische Denkmalschutzgesetz aufgenommen.

\subsection{Gesichts- und Geschichtsverlust - kritische Stimmen um 1900}

Eine erste kritische Wahrnehmung der Veränderung des Wiener Stadtbildes ist bereits in der Zeit des Reformabsolutismus und Vormärz zu verzeichnen. ${ }^{83}$ Zwischen 1770 und 1830 entwickelte die Bevölkerung eine Begeisterung für die wachsende Großstadt, die 
mit einer zunehmenden Wertschätzung der Altstadt und einem kritischen Blick auf die Veränderung der Stadt einherging, so Sándor Békési. ${ }^{\mathbf{8 4}}$ Erste Anzeichen für das Bedürfnis nach der Erhaltung der „alten Stadt“ und das Interesse an ihrer Geschichte keimte in Wien also bereits im auslaufenden 18. Jahrhundert. Um 1800 setzte sich schließlich eine neue, kritische Sicht der sich allzu rasch vollziehenden Stadterneuerung durch, die nicht nur auf die baulichen Veränderungen, sondern, so Békési, vor allem auf die sozialen, wirtschaftlichen und politischen Umwälzungen zurückzuführen sei. Die Geschichtsschreibung begann sich mit der Stadt und ihrer Baugeschichte zu befassen und man versuchte zunehmend, das Alte und Verschwindende zu dokumentieren. ${ }^{\mathbf{8 5}}$ Eine strikte Ablehnung der Stadterneuerung und eine nostalgisch-emotionale Besetzung der verschwindenden Stadt mit der gleichzeitigen Verwendung des Begriffs „Alt-Wien“ zeigt sich erstmals in den Schriften des Stadtchronisten Franz Gräffer. ${ }^{\mathbf{8 6}}$

Verlustangst und „Alt-Wien-Nostalgie“ entstanden also nicht erst als eine Folge der gründerzeitlichen Demolierungswelle, sondern bereits deutlich früher. Ausdruck fand diese nostalgische Stadtwahrnehmung, die Sehnsucht nach vergangenen Zeiten und verlorenen Stadträumen, unter anderem in spätbiedermeierlichen Stadtansichten $^{\mathbf{8 7}}$ und melancholischen Wienerliedern ${ }^{\mathbf{8 8}}$. Die biedermeierliche Kunst entdeckte vor allem das bürgerliche Wohnhaus als Motiv. Schon ab den 1820ern wurden in Sepiazeichnungen und Aquarellen erinnerungswürdige und abrissgefährdete Häuser dokumentarisch - zumeist aber mit verklärtem Blick - festgehalten. Die alten Häuser wurden zu Identitätsträgern und laut Békési zum „Medium des urbanen Gedächtnisses“, $\mathrm{zu}$ „Kristallisationspunkten der kollektiven Stadterinnerung“.89 Es entstand ein zum Teil idealisiertes Bild von „Alt-Wien“, das durch die gesellschaftlichen und wirtschaftlichen Umbrüche verloren zu gehen drohte. ${ }^{90}$

Parallel zu dieser zunehmend kritischen Wahrnehmung der rasanten baulichen Veränderung der Stadt entwickelte sich um 1900 das zuvor skizzierte denkmalpflegerische Interesse an „Alt-Wien“. Gegen den zunehmenden Verlust des historischen Stadtbildes erhoben sich gewichtige Stimmen. Kunsthistoriker und Denkmalpfleger, wie Max Dvořák, aber auch Architekten und Vertreter des Städtebaus, wie vor allem Camillo Sitte, beklagten in zahlreichen Publikationen die fortschreitende und scheinbar haltlose Zerstörung ganzer Altstadtbereiche und befassten sich gleichermaßen mit neu zu definierenden Richtlinien für den zukünftigen Städtebau. War über Jahrhunderte „trotz der größten wirtschaftlichen Umwälzungen [...], trotz der größten technischen und künstlerischen Neuerungen [...] nur modifiziert und weiterentwickelt" und die Stadt mehr oder minder behutsam adaptiert worden, habe man vor allem ab der Mitte des 19. Jahrhunderts damit begonnen, die historisch gewachsenen Altstädte an die neuen Ansprüche und Anforderungen anzupassen, wie Dvořák 1910 kritisch festhält.91 Und auch im bereits erwähnten 15. Band der Österreichischen Kunsttopographie von 1916 erhebt er mahnende Worte:

„Unheimlich schnell verschwindet das alte Stadtbild von Wien und mit ihm eines der wichtigsten Zeugnisse der allmählichen Entstehung und Entwicklung der Stadt. Eine kartographische Aufnahme vermag heute noch Zusammenhänge festzuhalten, von denen in wenigen Jahren nichts oder nur ein Rest von zusammenhanglosen Gliedern vorhanden sein wird.“92 
Dieses Zitat zeigt deutlich, dass die Besorgnis um den Verlust der über Jahrhunderte geformten Wiener Altstadt den denkmalpflegerischen Diskurs bereits im frühen 20. Jahrhundert kennzeichnete. Der zunehmende „Substanzverlust“ sorgte letztlich auch für die Aufnahme Wiens in die Reihe der Denkmalinventare.93 Die für diesen Band erstellten „Kunsthistorischen Pläne“, die Max Dvořák als „wichtigen Behelf der praktischen Denkmalpflege z. B. bei Regulierungsfragen“ 94 bezeichnete, vermochten es dabei, den abstrakten Begriff „Alt-Wien“ anschaulich zu machen. Unter „Alt-Wien“ verstand man damals die Ensembles aus Bürgerhäusern des 16. und 17. Jahrhunderts und herrschaftlichen Palais des Barock und Klassizismus, die in Biedermeierhäusern bescheidene Begleitung fanden und die in der Innenstadt - farbig in der Karte herausgestellt - „inselartig“ zwischen den später überformten Bereichen vorzufinden waren (Abb. 7). In den äußeren Bezirken waren es die verbliebenen Strukturen der ehemaligen Vororte, die die „Einverleibung“ in die Großstadt überdauert hatten. Deutlich erkennen lässt die Karte zum 1. Bezirk, dass sich diese Ensembles als eine Folge der gründerzeitlichen Stadterneuerung auf die Bereiche abseits der Hauptgeschäftsstraßen (Kärntner Straße, Graben, Rotenturmstraße) zurückgezogen hatten. Gebäude, die vor 1850 errichtet worden waren, wurden nicht farbig kartiert, wodurch gleichsam der bedeutsame Bestand vom weniger erhaltenswerten geschieden wurde.

Ähnlich wie Dvořák verweist auch Hans Tietze, ebenfalls Kunsthistoriker, Universitätsprofessor und Kollege Dvořáks in der Zentralstelle für Denkmalschutz, auf den sich unglaublich rasch vollziehenden „Verstädterungsprozess“ in den ehemaligen Vorstädten: „,...] wo sich gestern Felder dehnten, strecken sich morgen ungeheure Arbeiterviertel aus, deren gerade und schematisch gezogenen Zinshäuserzeilen die Individualität der alten Ortschaften niederstampfen. Die alten, natürlich gebogenen Dorfstraßen werden in die gerade Linie gezwängt, die Kirchlein, die für die verhundertfachte Bevölkerung viel zu klein geworden sind, weichen ungeheuren Neubauten.“95

Dass auch die Architektur- und Stadtfotografie gerade um 1900 in Wien „boomte“, ist Indiz für das in der Öffentlichkeit angekommene Interesse an den baulichen Veränderungen und dem drohenden Verlust charakteristischer Altstadtbereiche. August Staudas fotografische Dokumentation der Wiener Innenstadt und der Vororte ist als Versuch zu lesen, einen bedrohten baulichen Zustand der Stadt zumindest im schwarz-weißen Bild zu konservieren.96 Gerade die malerischen, verwinkelten Vororte und die dort oft ärmlichen Verhältnisse wurden in Staudas rund 3.000 Fotografien umfangreich dokumentiert. Interessant ist, dass gerade ein Denkmalpfleger als sein wichtigster Auftraggeber gilt: Graf Karl Lanckoroński-Brzezie, Sammler, Mäzen und ab 1910 Vizepräsident der Zentralkommission, beauftragte ihn als Privatmann mit zahlreichen Dokumentationen.97

Ausdruck des steigenden Interesses an der baulichen Vergangenheit der Stadt sind auch die im Jahr 1892 im Rahmen der Internationalen Ausstellung für Musik und Theaterwesen in Wien und 1893 bei der Weltausstellung in Chicago nahezu in Originalgröße errichteten, spätmittelalterlich anmutenden und teils willkürlich zusammengestellten „Alt-Wien“-Ensembles. Diese begehbaren Nachbildungen von in dieser Form nie dagewesenen Plätzen und ihrer angrenzenden Bebauung sollten allerdings weniger Kritik am Verlust von „Alt-Wien“ üben, als vielmehr der Unterhaltung der Besucher und als stimmungsvoller Rahmen für die Vermittlung österreichischer Kultur und die Anpreisung österreichischer Konsumgüter dienen.98 
Manche Fachleute sahen in diesen Jahren den Verlust „Alt-Wiens“ schon bedrohlich nahegekommen und „ganze Plätze und Stadtteile der Demolierungsmanie zum Opfer fallen“99. Camillo Sitte forderte in seinem einflussreichen Werk Der Städte-Bau nach seinen künstlerischen Grundsätzen von 1889 eine angemessene künftige Stadtplanung. ${ }^{100}$ Die erhaltenswerten Teile der Altstadt sollten dafür als „gebautes Lehrbuch“ für zukünftige Generationen erhalten bleiben. Sitte maß der historischen Stadt also neben einem gewichtigen ästhetischen und historischen Wert auch einen erzieherischen Wert bei. In einem Artikel im Neuen Wiener Tagblatt aus dem Jahr 1891 warnte er mit drastischen Worten vor der drohenden „Ausweidung Wiens“ und dem unüberlegten und daher zumeist unnötigen Niederreißen alter Gebäude unter dem Vorwand der verkehrstechnischen Notwendigkeit. ${ }^{101}$ Unsinnigerweise versuche man seit Jahren unter hohen Kosten in allen Teilen der Stadt gleichzeitig, ,ihre natürlich gewachsene, stromartige Verkehrsstruktur allmälig [sic!] in die moderne Schachbrettstruktur überzuführen“, anstatt an einzelnen Punkten überlegt „Straßenverbreiterungen und Geradrichtungen“ durchzuführen. ${ }^{\mathbf{1 0 2}}$ Sitte befürwortete also auch radikale städtebauliche Eingriffe, sofern sie zweckmäßig und durchdacht waren und in der Ausführung „dem bestehenden Alten“ um nichts nachstanden. Kritik übte er im Jahr 1893 am fehlenden Mut und an der gleichzeitigen „Prinzipienreiterei“ des Stadtbauamtes, das zahlreiche kleinteilige Lösungsversuche unternehme, anstatt einer großen künstlerischen Idee Raum zu geben. Wollte man das Verkehrsproblem lösen und die Innere Stadt dem Verkehr preisgeben, so müsse man eine radikale, aber ebenso durchdachte und künstlerisch hochwertige und „weltstädtische“ Idee umsetzen, beispielsweise in der Anlage einer monumentalen „Avenue ersten Ranges“ vom Praterstern durch die Innenstadt über den Graben bis nach Schönbrunn. ${ }^{\mathbf{1 0 3}}$ Kurze Zeit später sollte er, als einer der wenigen Fachleute des Städtebaus, Kritik an Karl Mayreders Regulierungsplan für den 1. Bezirk von 1895 üben, der zahlreiche radikale Durchbrüche und Begradigungen vorsah. ${ }^{\mathbf{1 0 4}}$ Wettbewerbsjurys sollten seiner Ansicht nach nicht aus Bürgermeister, Stadtbaudirektor und Gemeinderäten bestehen, sondern aus Fachleuten aus dem Ausland, wie etwa Camillo Boito, der „Venedig vor dem Fluche moderner Regulierung gerettet" habe. ${ }^{\mathbf{1 0 5}}$ Er verstand den Städtebau also vielmehr als künstlerische Disziplin denn als rein verkehrsplanerische und bautechnische Herausforderung. Gerade durch Sitte erlangte die historisch gewachsene Stadt im deutschen Raum eine erste echte Wertschätzung, wobei es Sitte vordergründig um die Verbesserung des zeitgenössischen Städtebaus ging, der von historischen Anschauungsbeispielen profitieren sollte, und weniger um die Erhaltung des historischen Stadtbildes per se. ${ }^{\mathbf{1 0 6}}$

In seinem Katechismus der Denkmalpflege benannte Max Dvořák, als wohl gewichtigste Stimme aus dem Bereich der Denkmalpflege, Gründe für die mutwillige Zerstörung des Stadtbildes: den „Fortschrittsgedanken“ und die bedingungslose Anpassung an die „Forderungen der Gegenwart“. Gleichermaßen verwies er auf die „Zerstörung alter Denkmäler aus falscher Verschönerungssucht“.107 Es sei eine „selbstverständliche Pflicht“, die Umgestaltung der Großstädte „nicht dem Zufall, den materiellen Interessen allein oder dem Gutdünken der gewöhnlichen Bauämter oder Verwaltungsorgane“ zu überlassen, sondern jenen Männern, „die mit allen nicht nur praktischen, sondern auch ästhetischen Erfordernissen des Städtebaues und den Rechten und Erfordernissen der Denkmalpflege" vertraut seien. ${ }^{\mathbf{1 0 8}}$ Verluste an Denkmälern und 


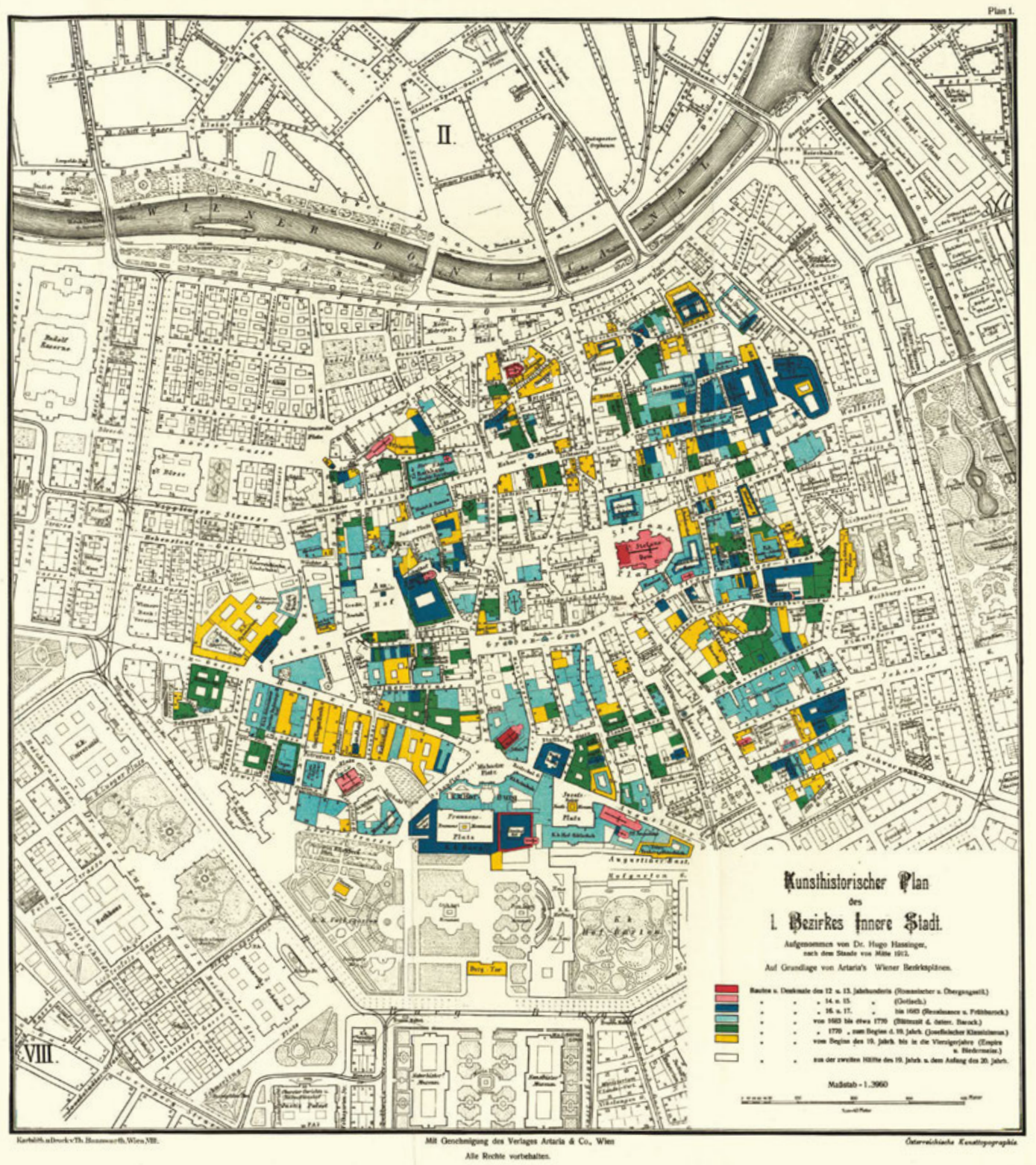

Abb. 7 Hugo Hassinger, „Kunsthistorischer Plan des 1. Bezirkes“, 1912

Altstadtbereichen bewertete er als Zeugnis für die „Unfähigkeit oder Leichtfertigkeit in der Stadtverwaltung “. ${ }^{\mathbf{1 0 9}}$ Damit spielte er sicherlich vor allem auf die in Wien vor sich gehende Entwicklung an. Die ästhetisch und städtebaulich heiklen Aufgaben der Stadtgestaltung sollten seiner Ansicht nach, ähnlich wie von Camillo Sitte gefordert, in die Hände befähigter und erfahrener Fachleute gelegt werden, um zu verhindern, dass „mit dem Lineal entworfene Straßen“ ganze Altstadtbereiche zerstörten. ${ }^{110}$ Ähnliches sollte wiederum Clemens Holzmeister im Jahr 1937 fordern, aber dazu später. ${ }^{111}$ Bereits 1908 verfasste Dvořák einen kritischen Kurztext zur verkehrstechnischen 
Regulierung Wiens, worin er vehement kritisierte, dass man „Alt-Wien“ ungerechtfertigten und nur scheinbar unumgänglichen „Verkehrsrücksichten“ opfere, unter „Beseitigung aller ,Engpässe‘ und Durchführung neuer Straßendurchbrechungen, denen die schönsten Partien des alten Wiener Stadtbildes zum Opfer fallen [...]" würden. ${ }^{\mathbf{1 1 2}}$ 1910 und 1911 äußerte er vehemente Kritik an der Stadtplanung der Gründerzeit, die sich an die Zerstörung des barocken Wien gemacht und rücksichtslos ganze Stadtteile zerstört habe:113

\begin{abstract}
„Nicht aus Böswilligkeit, sondern aus Überzeugung, daß es die Zeit, das Wohl und die Reputation der Stadt, der Freisinn und weite Blick ihrer Vertreter erfordere, begann man gegen das Alte zu wüten. [...] In der Flutwelle des Kampfes gegen die Überlieferung und für neue allgemeine Ideale, die sich nach der großen Revolution über Mitteleuropa ergoß, verlor man den Zusammenhang mit der heimatlichen künstlerischen Vergangenheit, ohne etwas an ihre Stelle setzen zu können, als eine ziellose und oft barbarische Neuerungssucht und Zerstörungsfreude."114
\end{abstract}

Ein Jahr später fand er noch drastischere Worte und kritisierte das durch „Spekulantentum [...], Gleichgültigkeit, Unwissenheit und Kunstblindheit“ verursachte „vandalische Vorgehen“, konstatierte jedoch auch einen endlich wachsenden Unmut in der Bevölkerung und forderte die Aussprache mit den bei der Gemeinde für die Regulierungspläne und Bauordnung zuständigen Personen. Er stellte eine neue „geistige Bewegung“ fest, die die Schönheit der alten Städte und Gassen wieder schätzte und nun endlich das zu schützen veranlasste, „was sich in den Städten noch an alten schönen Bauwerken, Gassen und Plätzen“ erhalten habe. ${ }^{\mathbf{1 1 5}}$

Auch Hans Tietze kritisierte 1910 die „fortwährende Modernisierung“ und radikale Umgestaltung der Stadt seit dem 19. Jahrhundert, durch die nun, nach Jahrhunderten des steten, aber gemäßigten, „natürlichen“ Wandels des Stadtbildes, der einzigartige Charakter Wiens völlig verloren zu gehen drohe. ${ }^{\mathbf{1 1 6}}$ Eine weitere kritische Stimme war jene von Hugo Hassinger117, Raumforscher und Kulturgeograf, der sämtliche, zuvor angesprochenen kunsthistorischen Stadtpläne für die Österreichische Kunsttopographie zu Wien von 1916 erstellte. ${ }^{\mathbf{1 1 8}}$ Hassinger forderte eindringlich, dem „Verderbnis des Stadtbildes“ und der „fortschreitenden Verflachung der Persönlichkeit Wiens“ nun endlich Einhalt zu gebieten. ${ }^{\mathbf{1 1 9}}$ Dafür nahm er die städtischen Magistrate in die Pflicht und nicht die Zentralkommission. ${ }^{\mathbf{1 2 0}}$ Wie viele andere sah auch er den veralteten Regulierungsplan des späten 19. Jahrhunderts, der allein verkehrstechnische Aspekte und somit den Grundriss der Stadt berücksichtige, auf den Aufriss und das „künstlerische Bild der Stadt" allerdings keine Rücksicht nehme, als Ursache für die anhaltende Zerstörung. 121

Erwähnt werden muss an dieser Stelle auch der 1871 in Wien geborene Kunstschriftsteller und „Kulturaktivist“122 Joseph August Lux, der sich sowohl in Tageszeitungen zu Umbauplänen äußerte als auch eigenständige Publikationen zum Thema Städtebau verfasste. ${ }^{\mathbf{1 2 3}}$ Lux war kurz vor der Jahrhundertwende nach England gegangen, wo er die Arts-and-Crafts-Bewegung rezipiert hatte, vor allem die Schriften von John Ruskin und William Morris. Lux wird in der Literatur, auch durch seine kritische Haltung gegenüber dem Historismus und seine Wiederentdeckung des Biedermeier, als ein Vorreiter der österreichischen Heimatschutzbewegung bezeich- 
net. ${ }^{\mathbf{1 2 4}}$ Einhergehend mit der Kritik an Architektur und Stadtumbau der Gründerzeit galt um 1900 das Biedermeier, die Zeit vor dem dekadenten, eklektischen Bauboom, getragen von den Bodenspekulanten und dem Großbürgertum, als erstrebenswerte Ausdrucksform - sich auszeichnend in gestalterischer Zurückhaltung, Bescheidenheit und Materialechtheit.

Die steigende Wertschätzung des historischen Stadtbildes einerseits und die rege Publikationstätigkeit andererseits können als Reaktion auf den massiven Stadtumbauprozess in Wien im Laufe des 19. Jahrhunderts gelesen werden. ${ }^{\mathbf{1 2 5}}$ Radikale Bauvorhaben in der Wiener Innenstadt um 1900 und in den ersten Jahren des 20. Jahrhunderts befeuerten neuerlich die Diskussionen um den Erhalt von „Alt-Wien“. Dabei gab es auch Fälle, die sowohl die Architektenschaft als auch das Stadtbauamt und die institutionelle Denkmalpflege an einem Strang ziehen ließen, nämlich dann, wenn alle Parteien eine Maßnahme für nicht zweckmäßig und für das Stadtbild nicht zuträglich erachteten. So verurteilte beispielsweise die Zentralstelle ebenso wie Karl Mayreder den Anfang 1912 durchgeführten Straßendurchbruch vom Graben zur Goldschmiedgasse über den Grund des Trattnerhofes ${ }^{\mathbf{1 2 6}}$ und plädierte für die Erhaltung der geschlossenen Platzwand. Die Denkmalpflege äußerte sich also nicht nur kritisch bei Fragen der Regulierung und des Abbruchs, sondern auch bei Gestaltungsfragen. ${ }^{\mathbf{1 2 7}}$

Das Verhältnis von „Alt“ und „Neu“ und die Frage der Einbindung von Neubauten in den historisch gewachsenen baulichen Kontext stellten seit dem frühen 20. Jahrhundert ein zentrales Thema im Diskurs der Denkmalpflege dar. So forderte Max Dvořák, auch neue Architektur und einen „zeitgemäßen Stil“ unter Rücksicht auf „das schöne Alte“ zuzulassen. Retten solle man hingegen, „was bloßem Mutwillen, unsolider Spekulation oder purem Unverstand“ geopfert werden sollte. ${ }^{\mathbf{1 2 8}}$ Die Denkmalpflege war an der Erhaltung der Struktur und des Bildes der Stadt interessiert, betrachtete damals aber auch die Gestaltung von Neubauten im historischen Umfeld als ihre Aufgabe und war daher eng verknüpft mit Fragen der zeitgenössischen Stadtgestaltung. ${ }^{\mathbf{1 2 9}}$ Nicht nur Kunsthistoriker, sondern auch viele Städtebauer und Architekten waren der Ansicht, dass sich Neubauten in die bestehende Struktur und das Gesamtbild der Stadt einfügen mussten. Der Abriss von Bestandsbauten war legitim, allerdings nicht bedingungslos zu befürworten. Wertvolle Baudenkmale sollten unter allen Umständen erhalten bleiben.

In Übereinstimmung damit erachtete auch die Heimatschutzbewegung gewisse „materielle Opfer" als durchaus vertretbar und forderte einen angemessenen, natürlichen Prozess der Stadtveränderung. Das heimische Bauwesen sollte dabei weiterentwickelt werden und die zeitgenössische Architektur ihren Platz finden. Die „neuen Schöpfungen“ sollten laut Tietze allerdings „mit den bestehenden Monumenten in Einklang" gebracht werden und hatten nur ihre Berechtigung, sofern sich der Abbruch des Altbestandes tatsächlich als notwendig erwies. ${ }^{\mathbf{1 3 0}}$

Vergleichbare Töne schlägt Hassinger 1912 in seinem Plädoyer für die Heimatschutzbewegung an. Sich auf Paul Schultze-Naumburg berufend, sei es nicht Absicht des Heimatschutzes, „die Dinge museal zu konservieren“:

„Die Heimatschutzbestrebungen wollen nicht um jeden Preis das Alte erhalten, aber sie fordern, daß in jedem einzelnen Falle, wo es gilt, ein altes Stück unserer Heimat 
durch ein neues zu ersetzen, geprüft werde, ob es das alte, in seinem Kulturwert gleich- und höherwertig, zu ersetzen vermag. Sie fordern, daß sich Neues harmonisch unserer alten Heimat einfüge, nicht aber, daß in gedankenloser Verfolgung doktrinärer Schlagworte von ,Fortschritt, Verkehr, Hygiene‘ blindlings das Alte geopfert werde." 131

Vielfach wurde demzufolge bereits um 1900 der Gedanke des Ensembles aufgeworfen, wenn auch der Begriff noch keine explizite Erwähnung fand. Bei Dvořák wird schließlich der Schutz historisch gewachsener Altstadtbereiche als wesentliches Ziel der Denkmalpflege definiert, ja gerade der das Denkmal umgebenden Bebauung wird eine besondere Bedeutung zugeschrieben. ${ }^{\mathbf{1 3 2}}$ Unüberlegte, dem Verkehr geschuldete „Verluste und Verwüstungen zu verhindern“, verstand er als zentrale Aufgabe des Denkmalschutzes. ${ }^{133}$

Durch den Ausbruch des Ersten Weltkrieges wurde der umfangreiche Stadtumbauprozess vorerst verlangsamt. In den folgenden 1920er-Jahren beschränkte sich die stadtplanerische Tätigkeit der Gemeinde Wien vor allem auf die Wohnbauoffensive und die Errichtung zahlreicher Gemeindebauten, angesiedelt zumeist an der Peripherie der Stadt. Massive Eingriffe in die Altstadtstruktur in diesen Jahren blieben weitgehend aus. Vermutlich auch deshalb ebbt die Diskussion von Regulierungsfragen in den Tageszeitungen ab und sinkt die Zahl der Mahnschriften und Appelle der Heimatschützer und Denkmalpfleger. Themen der Altstadterhaltung ebenso wie städtebauliche Gestaltungsfragen werden weiterhin diskutiert, fortan jedoch vor allem in Architekturzeitschriften und denkmalfachlichen Periodika. ${ }^{134}$ 
1 Zur Stadtregulierung im 19. Jh. sind zahlreiche Publikationen erschienen. Einen guten Überblick über die Regulierungstätigkeit in diversen europäischen Haupt- bzw. Großstädten (darunter Brüssel, Straßburg, Berlin, Paris, Wien und Rom) bietet der Sammelband: Gerhard Fehl, Juan Rodríguez-Lores (Hrsg.), Stadt-Umbau. Die planmäBige Erneuerung europäischer Großstädte zwischen Wiener Kongreß und Weimarer Republik, Basel 1995. 2 Zur Herleitung und Bedeutung des Themas der „Hygiene“ im Städtebau siehe: Philipp Sarasin, „Die moderne Stadt als hygienisches Projekt. Zum Konzept der ,Assanierung“ der Städte im Europa des 19. Jahrhunderts“, in: Vittorio Magnago Lampugnani, Stadt \& Text. Zur Ideengeschichte des Städtebaus im Spiegel theoretischer Schriften seit dem 18. Jahrhundert, Berlin 2011, S. 99-112. Die städtebaulich technischen und auch medizinisch-hygienischen Aspekte der Assanierung sind bereits zu lesen bei: Theodor Weyl (Hrsg.), Die Assanierung von Wien, Leipzig 1902. Die Publikation geht auf die Auswirkungen der Assanierung auf Infektionskrankheiten und die Abnahme der Mortalität ein (bspw. durch die verbesserte Wasserversorgung, Kanalisation und auch durch Regulierungsmaßnahmen). 3 Renate Banik-Schweitzer, ,,Zugleich ist auch bei der Stadterweiterung die Regulierung der innern Stadt im Auge zu behalten. 'Wiener Altstadt und Ringstraße im Tertiärisierungsprozeß des 19. Jahrhunderts“, in: Gerhard Fehl, Juan Rodríguez-Lores (Hrsg.), Stadt-Umbau. Die planmäßige Erneuerung europäischer Großstädte zwischen Wiener Kongreß und Weimarer Republik, Basel 1995, S. 127-147, hier S. 142. 4 Einen kurzen Überblick über die Stadtsanierung und "Stadtgesundung“ vom 19. Jh. bis nach dem Zweiten Weltkrieg bietet: DieterJürgen Mehlhorn, Städtebau zwischen Feuersbrunst und Denkmalschutz. Erhaltung - Veränderung - Bewahrung, Berlin 2012, S. 257-260. 5 Zu den Umbaumaßnahmen in der Inneren Stadt in der 2. Hälfte des 19. Jh. siehe u. a.: Renata Kassal-Mikula, „Alt-Wien unter dem Demolierungskrampen. Wiens Innenstadt nach 1858“, in: Kos/Rapp 2005, S. 46-61. 6 Zum Stadtumbau in dieser Zeitspanne und auch zur Wahrnehmung der Veränderung des Stadtbildes siehe: Sàndor Békési, „Zwischen Alt und Neu. Umbau und Wahrnehmung von Wien um 1800", in: Karl Brunner, Petra Schneider (Hrsg.), Umwelt Stadt. Geschich te des Natur- und Lebensraumes Wien (Ausstellungskatalog, Wien Museum), Wien u.a. 2005b, S. 574-583. 7 Als Citybildung, auch Tertiärisierung der Innenstadt genannt, bezeichnet man die Konzentration der Wirtschaft und des Handels im Zentrum und die Verdrängung der Wohnfunk tion, meist einhergehend mit einer zumindest in Teilen sich vollziehenden „Verslumung“ ganzer Altstadtbereiche; siehe dazu u.a.: Ruth Hanisch, „Die formative Kraft des Faktischen. Erweiterung und Modernisierung der Großstadt“, in: Vittorio Magnago Lampugnani, Katia Frey, Eliana Perotti (Hrsg.), Anthologie zum Städtebau, 5 Bde., Bd. 2.1, Das Phänomen Großstadt und die Entstehung der Stadt der Moderne, Berlin 2014, S. 7-24, hier S.19. 8 Banik Schweitzer 1995, S.144-146. 9 Ebd., S.131; siehe dazu auch: Hans Bobek, Elisabeth Lichtenberger, Wien. Bau liche Gestalt und Entwicklung seit der Mitte des 19. Jahrhunderts (Schriften der Kommission für Raumforschung der ÖAW, 1), Wien u.a. 1978, S. 95 f. 10 Für die Zurück- verlegung der Baulinie und die entstandene Straßenfläche musste die Gemeinde entsprechende Entschädigungen zahlen. Auch die Zusammenlegung und Beschneidung der Parzellen und die anschließende Versteigerung brachten der Gemeinde herbe Verluste ein, weshalb man die Altstadtsanierung wieder in die Hände privater Investoren und der nun entstandenen Baugesellschaften legte. Siehe: Banik-Schweitzer 1995, S.126-147. Auf die schlechte finanzielle Lage der Gemeinde und die dadurch nicht - so wie in Paris - mögliche, bewundernswerte, „energische Methode der Stadtregulierung“ verweist bereits Heinrich Goldemund in: ders., „Die bauliche Entwicklung und Stadtregulierung“, in: Weyl 1902, S. 109. 11 Harald Bodenschatz, „Abbruch und Erhalt. 150 Jahre Planungsgeschichte der europäischen Altstadt“, in: Forum Stadt, 46. Jg., H. 2 , 2019, S. 113-133, hier S. 116. 12 Karl Ginhart, „Kurzberichte. Wien“, in: Deutsche Kunst und Denkmalpflege, 1936, S. 303-305, hier S. 304. 13 Adalbert Klaar erstellte bereits ab 1931 Bauaufnahmen und Baualterpläne v. a. von Bauernhäusern in Salzburg, Niederösterreich und Oberösterreich. Während der NS-Herrschaft war er in der Planungsbehörde der „Reichsstelle für Raumordnung in Wien“ tätig. Ab 1946 fertigte er für das Bundesdenkmalamt Baualterpläne zahlreicher österreichischer Städte an. Siehe: Theodor Brückler, Ulrike Nimeth, Personenlexikon zur österreichischen Denkmalpflege, Wien 2001, S. 134-135. 14 Adalbert Klaar, „Umwandlung des Wiener Stadtbildes“, in: Jahrbuch des Vereins für Geschichte der Stadt Wien, Bd. 12, 1955/56, S. 182-197, hier S. 192-194. 15 Goldemund 1902, S.108. 16 Heinrich Goldemund, "Städtebauliche Aufgaben Wiens in der nächsten Zukunft", in: Festschrift des Österr. Ingenieur- und Architektenvereines, Wien 1923, S. 23-29, hier S.37. 17 Vgl. Gottfried Pirhofer, Kurt Stimmer, Pläne für Wien. Theorie und Praxis der Wiener Stadtplanung von 1945 bis 2005, Wien 2007, S. 13-20. 18 Wolfgang Mayer, Verein für Geschichte der Stadt Wien (Hrsg.), Die städtebauliche Entwicklung Wiens bis 1945 (Ausstellungskatalog, Wiener Stadt- und Landesarchiv), Wien 1978, S.18-19. 19 Max Dvořák im Vorwort in: K.K. Zentral-Kommission für Kunst- und Historische Denkmale (Hrsg.), Österreichische Kunsttopographie, Bd. 2, Die Denkmale der Stadt Wien (XI.-XXI. Bezirk), Wien 1908, S. V. 20 Wichtige Erkenntnisse zu den Zusammenhängen zwischen Stadtbild und Bauvorschriften liefert Harald Stühlinger in: ders., ,'Der Anstrich des Gebäudes muss den Augen unschädlich seyn.' Die Bauordnungen des 19. Jhdts. und ihre Auswirkungen auf Stadtbild und Stadtgestalt von Wien“, in: dérivé, H. 31, 2008, S. 54-60. 21 Vgl. Renate Schweitzer, „Der Generalregulierungsplan für Wien (1893-1920)“, in: Österreichische Gesellschaft für Raumforschung und Raumplanung (Hrsg.), Berichte zur Raumforschung und Raumplanung, Bd. 14, 1970, S. 24-41, hier S. 32; siehe dazu: LGBI. Nr. 48/1890 für das Erzherzogthum Österreich unter der Enns (Gesetz vom 26. December 1890, womit einige Bestimmungen der Bauordnung für Wien abgeändert werden) und den neu eingeführten $\S 105$ Wirkungskreis des Gemeinderathes und des Stadtrahtes. 22 Zur Vorgeschichte und zur Erstellung des Generalregulierungsplanes siehe v.a.: Schweitzer 1970, S. 24-41. 23 Ebd., S. 25-27. 24 Mayer 1978, S. 27. 25 Schweitzer 1970, S. 32-33. 26 Ebd., S. 32. 
27 Dazu äußerte sich bereits Cornelius Gurlitt, Handbuch des Städtebaues, Berlin 1920, S. 255. Publiziert wurde der Plan Mayreders 1896 in: Karl Mayreder, Motiven-Bericht zum Antrage des Stadtbauamtes für den Regulirungsplan der Inneren Stadt Wien, Wien 1896. 28 Ebd., S. $6 \mathrm{f}$. 29 Hugo Hassinger, „Die Vernichtung des Wiener Stadtbildes“, in: Max Dvořák, Flugschriften des Vereines zum Schutze und zur Erhaltung der Kunstdenkmäler Wiens und Niederösterreichs, 6 Bde., Bd.2, Zur Rettung Alt-Wiens, Wien/Leipzig 1910, S. 41-46. 30 Banik-Schweitzer 1995, S.140. 31 Siehe dazu u.a. die Akten im Archiv des Bundesdenkmalamtes: BDA-Archiv, Topographische Materialien, Wien I. Bezirk, Profan Allg.; vgl. u. a. Ed. Pötzl, „Der bedrohte Franziskanerplatz", in: Dvořák 1910, S. 32-38. 32 K.K. Zentral-Kommission für Kunst- und Historische Denkmale (Hrsg.), Österreichische Kunsttopographie, Bd. 15, Kunsthistorischer Atlas der k. k. Reichshaupt- und Residenzstadt Wien und Verzeichnis der erhaltenswerten historischen, Kunst- und Naturdenkmale des Wiener Stadtbildes, Wien 1916. 33 Besonders anschaulich werden die Ausmaße der Regulierungstätigkeit im frühen 20. Jh. durch den Vergleich einzelner Straßenzüge in den Generalstadtplänen, die im Abstand von mehreren Jahren erstellt wurden. Siehe dazu: Wiener Stadt- und Landesarchiv (WStLA), Pläne und Karten, Sammelbestand P2.1.883 und P2.1.309. 34 Siehe dazu bspw. den Aktenbestand im Archiv des Bundesdenkmalamtes, Sammlung Top. Mat., Wien Profan, Bezirke I-XIX. 35 Ersichtlich aus den Akten des Bundesdenkmalamtes, Aktenbestand wie in Anm. 34 angeführt (betrifft in der Sieveringer Straße die Hausnummern 11, 27, 89, 92-94, 95, 118-125, 172, 199-201, 251, 255) und anhand der entsprechenden Jahresausgaben des Amtsblattes der Stadt Wien. 36 Heinrich Strasser, Stadtbauamtsdirektion Wien (Hrsg.), Die Tätigkeit des Wiener Stadtbauamtes und der städtischen Unternehmungen technischer Richtung in der Zeit von 1935 bis 1965, 2 Bde., Bd. 1, Wien 1974, Abschnitt II, S. 9-10. 37 Laut Braumann kann der Stand der österreichischen Stadtplanung und des Städtebaus bis 1938 im internationalen Vergleich grundsätzlich als „rückständig“, aber dennoch oder gerade - aufgrund der schlechten wirtschaftlichen und der politischen Voraussetzungen als „beachtenswert" bezeichnet werden. Christoph Braumann, Stadtplanung in Österreich von 1918 bis 1945. Unter besonderer Berücksichtigung der Stadt Salzburg (Schriftenreihe des Institutes für Städtebau, Raumplanung und Raumordnung, TU Wien, 21), Wien 1986, S.12. 38 Befreiung von der „Hauszins- und Hausclassensteuer“; vgl. RGBI. 39/1880. 39 Vgl. dazu RGBI. 54/1893; It. Mayer kam das neue Gesetz beim Um- bzw. Neubau von 1.263 Liegenschaften zum Tragen; vgl. Mayer 1978, S. 21. 40 Pirhofer/Stimmer 2007, S. 18-19. 41 Bauordnung für Wien in der Fassung vom 25.11.1929, LGBL. für Wien 11/1930, III. Abschnitt, §§ 39-46. Der entsprechende Abschnitt entsprach damit dem Bundesgesetz vom Juni 1929 betreffend die Enteignung zu Wohn- und Assanierungszwecken; BGBI. 202/1929. 42 BGBI. 202/1929, § 2 Abs.1. 43 Siehe dazu den Kommentar zur Bauordnung von 1929: Richard Wolf (Hrsg.), Die Bauordnung für Wien. Mit einer Einleitung, erläuternden Bemerkungen, Auszügen aus Vorlage und Kommissionsbericht, einschlägigen anderen
Gesetzen und Entscheidungen des Verwaltungsgerichtshofes (Handausgabe österreichischer Gesetze und Verordnungen), Wien 1930, S. 76-77. 44 Siehe dazu: BGBI. 200/1929 und LGBI. für Wien 20/1929. 45 Bspw. wurde für die Objekte Billrothstraße 60-62 (1190) bereits 1925 der erste Abbruchantrag gestellt, 1933 erfolgte ein weiterer. Tatsächlich abgebrochen wurden die Gebäude im Jahr 1936 mit einer Förderung durch den Wiener Assanierungsfonds. Siehe dazu die Akten in: BDA-Archiv, Top. Mat., Wien XIX. Bezirk, Profan 1, Billrothstraße 60-62. 46 Auf die Verbindung der Disziplinen Stadtplanung und Denkmalpflege in ihrer frühen Entstehungsphase gegen Ende des 19. Jh. verweist u. a. Hans-Rudolf Meier, „Denkmalschutz als Leitinstrument der Stadtentwicklung ", in: Forum Stadt, H. 1, 2013, S. 35-51, hier S. 36; ders., ,Denkmalpflege und Stadtentwicklung in historischer Dimension“, in: Bundestransferstelle Städtebaulicher Denkmalschutz (Hrsg.), Historisches Erbe als Ausgangspunkt integrierter Stadtentwicklung, Berlin 2014, S. 10-15, hier S. 10. 47 Auf den Zusammenhang zwischen dem Stadtumbau des 19. Jhs. und der Entwicklung der Denkmalpflege verweist bereits Max Dvořák in: ders., ,,Denkmalpflege', Vortrag, gehalten am Denkmalpflegetag in Bregenz im Jahre 1920“, in: Mitteilungen des Bundesdenkmalamtes, 1924, S. 93-97, hier S. 96.48 Siehe dazu u.a.: Achim Hubel, Denkmalpflege. Geschichte, Themen, Aufgaben. Eine Einführung, Stuttgart 2019, S.100-105; Udo Mainzer, „Die Stadt und die Denkmalpflege“, in: Hans-Rudolf Meier, DENKmaIWERTE. Beiträge zur Theorie und Aktualität der Denkmalpflege, Berlin u. a. 2010, S. 191-202, hier S. 191-192. 49 Volkmar Eidloth, Heinrich Walgern, Gerhard Ongyerth (Hrsg.), Handbuch Städtebauliche Denkmalpflege (Berichte zu Forschung und Praxis der Denkmalpflege in Deutschland, 18), Petersberg 2013, S. 25-26. 50 Siehe dazu bspw.: Hubel 2019, S.103-104. 51 Siehe dazu: Marion Wohlleben, „Stadtbild - Oberfläche - Schein“, in: Sigrid Brandt, Hans-Rudolf Meier (Hrsg.), Stadtbild und Denkmalpflege. Konstruktion und Rezeption von Bildern der Stadt (Stadtentwicklung und Denkmalpflege, 11), Berlin 2008, S. 150-161, hier S. 158; Ingrid Scheurmann, Stadtbild in der Denkmalpflege: Begriff - Kontext - Programm, in: Brandt/ Meier 2008, S. 140-149, hier S.141. 52 Gerhard Vinken, Zone Heimat. Altstadt im modernen Städtebau, Berlin 2010, S. 7. 53 Karl Kühn, Die Schöne Altstadt. Ihr Schutz, ihr Umbau, ihre Verkehrsverbesserung. Eine Untersuchung zur praktischen Auswertung der Erkenntnis vom Wesen der alten Stadt, Berlin 1932, S. 5. 54 Bis zum Jahr 1910 fanden die Fachtagungen unter dieser Bezeichnung statt, ab 1911 wurden sie im Zweijahresrhythmus als Tag für Denkmalpflege und Heimatschutz abgehalten, allerdings mit Unterbrechung durch den Ersten Weltkrieg. Erst 1920 wurde die insgesamt dritte Tagung unter Beteiligung beider Organisationen abgehalten. Siehe dazu: Marion Wohlleben, Konservieren oder restaurieren? Zur Diskussion über Aufgaben, Ziele und Probleme der Denkmalpflege um die Jahrhundertwende (Veröffentlichungen des Instituts für Denkmalpflege an der ETH Zürich, 7), Zürich 1989, S. 37. 55 Auf die „wiederholte Beratung“ von Fragen der Altstadterhaltung seit dem ersten „Tag für Denkmalpflege“ im Jahr 1900 verweist u. a.: Gurlitt 1920, S. 272. Zur Stadterhaltungsdebatte um 1900 in der Denkmalpflege vgl. 
Melchior Fischli, „Die Restaurierung der Stadt. Stadtmorphologische Denkfiguren in der deutschen Altstadtdebatte um 1900“, in: Carmen Enss, Gerhard Vinken (Hrsg.), Produkt Altstadt. Historische Stadtzentren in Städtebau und Denkmalpflege (Urban Studies), Bielefeld 2016, S. 43-57. 56 Carl Rehorst, „Über die Möglichkeit der Erhaltung alter Städtebilder unter Berücksichtigung moderner Verkehrsanforderungen. Mannheim 1907“, in: Adolf von Oechelhaeuser, Denkmalpflege. Auszug aus den stenographischen Berichten des Tages für Denkmalpflege, Bd. 1, Leipzig 1910, S. 404-445, hier S. 404. 57 Paul Jonas Meier, „Über die Erhaltung alter Straßennamen. Bamberg 1905“, in: Oechelhaeuser 1910, S. 387-397, hier S. 387. 58 Siehe dazu das Programm in: Oechelhaeuser 1910. 59 Ein Vortrag zum damals höchst aktuellen Thema „Der Kampf um Alt-Wien“, den Josef Neuwirth, Professor für Kunstgeschichte an der Technischen Hochschule Wien $(\mathrm{TH})$, halten sollte, musste abgesagt werden. Stattdessen referierte Hugo Hassinger über die kartografische Aufnahme der Stadt Wien; Gemeinsame Tagung für Denkmalpflege und Heimatschutz. Salzburg 14. und 15. September 1911, stenographischer Bericht, Berlin 1911, S. 50 bzw. S. 123-126. 60 Siehe dazu das Kapitel „Der Plan des I. Bezirkes. a) Analyse des Kartenbildes“ in: K.K. Zentralkommission für Kunst- und Historische Denkmale, 1916, S. 37-48, v. a. S. 43-48. 61 Tag für Denkmalpflege und Heimatschutz. Würzburg und Nürnberg 1928. Tagungsbericht mit Sonderbeiträgen zur Heimat- und Kunstgeschichte Frankens, Berlin 1929. 62 Harald Bodenschatz, „Die historische Stadt im Visier der, Allgemeinen Städtebauausstellung in Berlin' 1910", in: Forum Stadt, H. 1, 2011b, S. 51-69, hier S.51. 63 Siehe eine Auflistung der Exponate in: Ernst Wasmuth (Hrsg.), Führer durch die allgemeine Städtebauausstellung in Berlin 1910, Berlin 1910. 64 Ebd., S. 5-19. 65 Bodenschatz 2011b, S. 59-60. 66 Siehe dazu: Gurlitt 1920; Otto Schilling, Innere Stadt Erweiterung, Berlin 1921. Letzterer vergleicht Sanierungsund Regulierungsmaßnahmen in mehreren mittel- und westeuropäischen Städten. $\mathbf{6 7}$ Eine vorwiegend durch ästhetische Überlegungen motivierte Maßnahme war die schon um 1800 einsetzende Freilegung bedeutender Bauwerke von den umliegenden oder angrenzenden baulichen Strukturen, um innen mit den dadurch entstehenden Plätzen und Straßenräumen zu einer gesteigerten Wirkung zu verhelfen. Békési 2005b, S.576. 68 Wolfgang Sonne, „Stadtbaukunst. Die Disziplin Städtebau als kulturelles Projekt“, in: Vittorio Magnago Lampugnani, Katia Frey, Eliana Perotti (Hrsg.), Anthologie zum Städtebau, 5 Bde., Bd. 2.1, Das Phänomen Großstadt und die Entstehung der Stadt der Moderne, Berlin 2014, S. 93-109. 69 Wolfgang Sonne, „Stadterhaltung und Stadtgestaltung. Schönheit als Aufgabe der städtebaulichen Denkmalpflege“, in: Hans-Rudolf Meier (Hrsg.), Werte. Begründungen der Denkmalpflege in Geschichte und Gegenwart, Berlin 2013, S.158-179, hier S.161-165. 70 Zur Verbindung von Denkmalpflege und Heimatschutz siehe u.a.: Norbert Huse (Hrsg.), Denkmalpflege. Deutsche Texte aus drei Jahrhunderten, München 2006, S. 150-160; Verena Jakobi, „Die Heimatschutzbewegung und die Entdeckung des Ensembles“, in: Ingrid Scheurmann (Hrsg.), Zeitschichten erkennen und erhalten. Denkmalpflege in Deutsch land, München/Berlin 2005, S.120-123; Ernst Bacher, „150 Jahre Denkmalpflege in Österreich“, in: Österreichische Zeitschrift für Kunst und Denkmalpflege (ÖZKD), Sonderheft, 54. Jg., H. 4, 2000, S. 460-465, hier S. 462; Theodor Brückler, „Zur Geschichte der Österreichischen Heimatschutzbewegung“, in: ÖZKD, 43. Jg., H.3/4, 1989, S. 145-156, hier S.145-146. 71 Ebd., S. 146; zur Entstehung der Heimatschutzbewegung in Österreich siehe auch: Karl Giannoni, „Die zwölfte österreichische Bundestagung für Heimatpflege zu Hall in Tirol 1933“, in: Die Denkmalpflege, 7. Jg., 1933a, S. 191. 72 Diese Bezeichnung wählt Karl Giannoni im Jahr 1933. Vgl. dazu: ders. Grundfragen des Heimatschutzes, Wien 1933b, S. 6. 73 Brückler 1989, S. 145-146. 74 Karl Giannoni, „Entwickelung und Ziele des Heimatschutzes in Österreich", in: Gemeinsame Tagung für Denkmalpflege und Heimatschutz. Salzburg 14. und 15. September 1911, stenographischer Bericht, Berlin 1911a, S. 83-97, hier S. 84. 75 Karl Giannoni war der Wegbereiter und wohl entscheidendste Vertreter der österreichischen Heimatschutzbewegung von deren Beginn bis in die Zeit des Nationalsozialismus (zu dieser Einschätzung gelangt auch Brückler in: ders. 1989, S. 157). Siehe dazu v.a. noch Kap. 5.2.3. 76 Giannoni 1911a, S. 84. 77 Lt. Teilnahmeliste: Emmerich Siegris, Hans Tietze, Dagobert Frey und Karl Holey; siehe: Gemeinsame Tagung für Denkmalpflege und Heimatschutz. Salzburg 14. und 15. September 1911, 1911, S.3-25. 78 Theodor Brückler, „Vom Konsilium zum Imperium. Die Vorgeschichte der österreichischen Denkmalschutzgesetzgebung“, in: ÖZKD, 45. Jg., H. 3/4, 1991, S. 160-173, hier S. 163-165; siehe zum Thema auch: Bundesdenkmalamt (Hrsg.), Denkmalpflege in Österreich, Wien 1990, S.144. 79 Karl Holey, Flugschriften des Vereines zum Schutze und zur Erhaltung der Kunstdenkmäler Wiens und Niederösterreichs, 6 Bde., Bd.5, Ein Denkmalschutzgesetz für Österreich, Wien/ Leipzig 1911, S. 3. 80 BGBI. 533/1923. 81 Bacher 2000, S. 462. 82 Ebenso wie die Zeit um 1900 kennzeichnete die 1970er-Jahre ein wirtschaftlicher Aufschwung, der ein gesteigertes Baugeschehen und eine vermehrte Zerstörung historischer Bausubstanz zur Folge hatte. Wiederum als Reaktion darauf wandte sich die Denkmalpflege erneut und nochmals verstärkt dem Thema der Altstadterhaltung zu. Auf diese Parallelen weisen u. a. Marion Wohlleben und Hans-Rudolf Meier hin: Wohlleben 2008, S. 150; Meier 2014, S. 10-15. Das erste und damit beispielgebende Ortsbildschutzgesetz in Österreich ist das 1967 erlassene Salzburger Altstadterhaltungsgesetz, es folgten die Altstadterhaltungsnovelle 1972 in Wien, das Grazer Altstadterhaltungsgesetz 1974 und diverse Ortsbildschutzgesetze in den Bundesländern, wie das Stadt- und Ortsbildschutzgesetz (SOG) in Tirol im Jahr 1976. 83 Siehe dazu vor allem: Sándor Békési, „Alt-Wien oder die Vergänglichkeit der Stadt. Zur Entstehung einer urbanen Erinnerungskultur vor 1848“, in: Wolfgang Kos, Christian Rapp, Alt-Wien. Die Stadt, die niemals war (Ausstellungskatalog, Wien Museum im Künstlerhaus), Wien 2005a, S. 29-38; sowie auch: Sándor Békési, „Die Erfindung von ,Alt-Wien' oder: Stadterzählungen zwischen Pro- und Retrospektive“, in: Monika Sommer, Heidemarie Uhl (Hrsg.), Mythos Alt-Wien. Spannungsfelder urbaner Identitäten, Innsbruck 2009, S. 45-66. 84 Im späten 18. Jh. erschienen 
erste Stadtbeschreibungen und frühe Reiseliteratur zu Wien, siehe bspw.: Franz de Paula Gaheis, Beschreibung der auffallendsten Merkwürdigkeiten der Haupt- und Residenzstadt Wien, Wien 1793. Sechs Jahre später erschien die Beschreibung der damaligen Vorstädte (heute Bezirke innerhalb des Gürtels). 85 Békési 2005a, S. 33. Békési verweist in diesem Zusammenhang u. a. auf: Josef Hormayr, Wien, seine Geschicke und Denkwürdigkeiten, Wien 1823-1825. 86 Békési 2005a, S.35. Békési verweist hier auf: Franz Gräffer, Kleine Wiener Memoiren. Historische Novellen, Genrescenen, Fresken, Skizzen, Persönlichkeiten und Sachlichkeiten, Anecdoten und Curiosa, Visionen und Notizen zur Geschichte und Characteristic Wien's und der Wiener in älterer und neuerer Zeit, Wien 1845. 87 Siehe dazu v. a.: Békési 2005b. 88 Siehe dazu: Paul Mahringer, „A Viertel Wein mit Liebe g'spritzt. Der verklärte, weinselige Blick auf Alt-Wien“, in: Denkmal heute, 7. Jg., H. 2, 2015, S. 32-37; Eva-Maria Hois, Ernst Weber, ,.... doch die Zeiten sind dahin ...!'. Alt-Wien im Wienerlied“, in: Kos/Rapp 2005, S.134-141. 89 Békesi 2005a, S.35. 90 Békési 2005b, S.577-579. 91 Siehe dazu: Dvoŕák 1910, S.3-7. Bei den Flugschriften handelt es sich um eine Zusammenstellung kritischer Artikel aus den Jahren 1907-1909 zu damals aktuellen Demolierungsvorhaben und geplanten Umbauten in der Inneren Stadt, die in Tageszeitungen erschienen waren, verfasst von unterschiedlichen Autoren, darunter u.a. Hugo Hassinger und Graf Karl Lanckoroński. 92 So Max Dvořák in seinem Vorwort zur Österreichischen Kunsttopographie; siehe: K. K. Zentralkommission für Kunst- und Historische Denkmale, 1916, o.S. 93 Schon 1908 - somit einige Jahre bzw. Bände vor der Darstellung der herausragenden Bauten der Inneren Stadt - hatte man sich im 2. Band der Österreichischen Kunsttopographie mit den ehemaligen Wiener Vororten befasst, die in der Spätgründerzeit durch die Eingemeindung und die rasante Stadterweiterung in relativ kurzer Zeit mit der Stadt „verschmolzen“ und durch diesen Prozess in ihrer Beschaulichkeit und ihrem dörflichen Charakter ganz besonders bedroht waren. Siehe dazu auch: Eckhart Vancsa, „Die Großstadt als Denkmal: Beispiel Wien“, in: ÖZKD, 4. Jg., H. 1/2, 1989, S.1-6, hier S. 3. 94 So Max Dvořák im Vorwort zum 15. Bd. der Österreichischen Kunsttopographie von 1916, siehe: K. K. Zentralkommission für Kunst- und Historische Denkmale, 1916. 95 Hans Tietze, Flugschriften des Vereines zum Schutze und zur Erhaltung der Kunstdenkmäler Wiens und Niederösterreichs, 6 Bde., Bd. 4, Das Wiener Stadtbild, Wien 1910, S. 32. 96 Neben der Fotografie war auch die Vedutenmalerei um 1900 auf die Darstellung verwinkelter Altstadtbereiche fokussiert. Siehe dazu: Elke Doppler, „Die Jäger der verlorenen Schätze. Wiener Vedutenmalerei von 1870 bis 1910“, in: Kos/Rapp 2005, S. 123-133. 97 Susanne Winkler, „August Stauda. ,Spezialist in Aufnahmen von Architekturen, Häusern, Villen, Schlössern und Interieurs ...'., in: dies. (Hrsg.), August Stauda. Ein Dokumentarist des alten Wien (Ausstellungskatalog, Wien Museum), Wien 2004, S.7-10; vgl. auch: dies., „,Alt-Wien“ im Wien Museum. August Stauda und Karl Graf Lanckoroński“, in: Bogusław Dybaś, Anna Ziemlewska, Irmgard Nöbauer (Hrsg.), Karl Lanckoroński und seine Zeit, Wien 2014 S. 217-237; Brückler 1989, S. 150-151. 98 Siehe dazu:
Ursula Storch, „Alt-Wien dreidimensional. Die Altstadt als Themenpark“, in: Kos/Rapp 2005, S. 159-164; Elke Krasny, „Beschwingte Gelassenheit und vornehme Zurückhaltung. Alt-Wien auf Weltausstellungen“, in: ebd., S.165-171. 99 Max Dvořák, „Aufruf!“ [1911], in: Sandro Scarrocchia, Max Dvořák. Schriften zur Denkmalpflege. Gesammelt und kommentiert von Sandro Scarrocchia (Studien zu Denkmalschutz und Denkmalpflege, 22), Wien u.a. 2012, S. 430-431 [Erstabdruck in: Josef Neuwirth, Die Denkmalpflege im Rahmen der städtischen Verwaltung (AltWien, 1), Wien 1911, S.3-7]. Der Aufruf wurde insgesamt von 111 Personen unterfertigt. 100 Sonne 2013, S. 160. 101 Camillo Sitte, „Die Ausweidung Wiens“, in: Neues Wiener Tagblatt, 6.12.1891, S.1-3. 102 Ebd., S. 2. 103 Camillo Sitte, „Der Wille des Stadtbauamtes“, in: Neues Wiener Tagblatt, 12.3.1893, S.1-3. 104 Schweitzer 1970, S. 34. 105 Sitte, 12.3.1893, S.3. 106 Sonne 2013, S.159-160. 107 Max Dvořák, Katechismus der Denkmalpflege, Wien 1918 [1. Auflage 1916], S.7. 108 Ebd., S. 50. 109 Ebd., S. 16: „Es ist die Pflicht der Stadtvertretungen, dafür zu sorgen und keine Opfer und Mühen zu scheuen, wo es sich um das Schicksal alter Bauten und Stadtteile handelt, denn auch für diese, nicht nur für technische Neuerungen sind sie verantwortlich und jedes ohne absolute Notwendigkeit geopferte Denkmal muß innen als Zeichen von Unfähigkeit oder Leichtfertigkeit in der Stadtverwaltung angerechnet werden." 110 Ebd. 111 WStLA, 1.5.4. Stadtbaudirektion, A1, Allg. Reg., Schachtel 109/1937, GZ 4353, Brief Clemens Holzmeister an Bgm. [Richard] Schmitz, 26.10.1937; siehe Kap. 3.1. 112 Max Dvořák, „Wiener Verkehrsrücksichten“ [1908], in: Scarrocchia 2012, S. 414-415, hier S. 414. 113 Vgl. dazu: Dvořák 1910; ders. [1911] 2012. 114 Dvořák 1910, S. 4-5. 115 Dvořák [1911] 2012, S. 430. 116 Tietze 1910, S.34-37. 117 In der NS-Zeit pflegte Hassinger vor allem interdisziplinäre Forschung, u. a. mit Hans Sedlmayr und Adalbert Klaar. Den Lehrstuhl für Kulturgeographie an der Universität Wien leitete er von 1931 bis 1951. Vgl. Petra Svatek, „Raumforschung an der Universität Wien im 20. Jahrhundert. Kontinuitäten und Wandlungen einer multidisziplinären und politisch orientierten Forschungsrichtung“, in: Katharina Kniefacz u. a., Universität - Forschung - Lehre. Themen und Perspektiven im langen 20. Jahrhundert (650 Jahre Universität Wien - Aufbruch ins neue Jahrhundert, 1), Wien 2015 , S. 241-259. 118 Hassinger nannte die „zunehmende Verwüstung und Vernichtung [des] ehrwürdigen Wiener Stadtbildes" als Auslöser für die Erstellung seiner kunsthistorischen Pläne. Siehe: Gemeinsame Tagung für Denkmalpflege und Heimatschutz. Salzburg 14. und 15. September 1911, 1911, S.123. 119 Hugo Hassinger, Wiener Heimatschutz- und Verkehrsfragen, Wien 1912, S. 16-20. 120 Ebd., S.10. 121 Ebd., S. 21; zur Kritik am Generalregulierungsplan siehe auch: S. 26. 122 Vittorio Magnago Lampugnani, Katia Frey, Eliana Perotti (Hrsg.), Anthologie zum Städtebau, 5 Bde., Bd. 2.1, Das Phänomen Großstadt und die Entstehung der Stadt der Moderne, Berlin 2014a, S. 136. 123 Joseph August Lux, Der Städtebau und die Grundpfeiler der heimischen Bauweise. Zum Verständnis für die Gebildeten aller Stände namentlich aber für Stadtverordnete, Baumeister, Architekten, Bauherren etc., Dresden 1908. 124 Siehe dazu: Ruth Hanisch, „Joseph August 
Lux und die Internationale der Heimatkunst“, in: Kai Krauskopf, Hans-Georg Lippert, Kerstin Zaschke (Hrsg.), Europäische Architektur im Zeichen von Traditionalismus und Regionalismus (Neue Tradition, 3), Dresden 2012 , S. 87-106. 125 So etwa die bereits mehrfach zitierten Flugschriften des Vereins zum Schutze und zur Erhaltung der Kunstdenkmäler Wiens und Niederösterreichs aus den ersten Jahren des 20. Jhs.; vgl. dazu auch die Bände Tietze 1910; Holey 1911; Karl Giannoni, Flugschriften des Vereines zum Schutze und zur Erhaltung der Kunstdenk mäler Wiens und Niederösterreichs, 6 Bde., Bd. 6, Heimatschutz, Wien/Leipzig 1911b. 126 Der „Fall Trattnerhof“ war wohl einer der am meisten diskutierten Fälle der Jahre vor dem Ersten Weltkrieg. Siehe dazu: „Der Straßendurchbruch beim Trattnerhof", in: Neue Freie Presse, 2.2.1912, S.10. 127 Siehe dazu zahlreiche Artikel in Tageszeitungen; bspw. „Die Straße durch den Trattnerhof“, in: Neues Wiener Tagblatt, 31.1.1912, S. 6; sowie in Fach- zeitschriften wie z.B.: „Vereinsberichte. Der Österreichische Ingenieur- und Architektenverein“, in: Der Bautechniker, 2.2.1912, S.10-11. 128 Dvoŕák [1911] 2012, S. 430-431. 129 Sonne 2013, S.161-165. 130 Tietze 1910, S. 43-45; „Die Freunde von Wien erheben Einspruch dagegen, daß ohne Not zerstört wird, daß Teile, die im Bilde Wiens unentbehrlich sind, vernichtet werden, weil bureaukratische Gesinnung und eine sich modern gebärdende Großstadtsucht sich weigern, ihre schematisch und längst unmodern gewordenen Anschauungen zu überprüfen“ (ebd., S. 45-46). 131 Hassinger 1912, S. 12-13. 132 In seinem Entwurf für ein österreichisches Denkmalschutzgesetz von 1911 unterstreicht Karl Holey ebenfalls die Bedeutung der Umgebung eines Denkmals, dessen Wirkung im Stadtbild erst durch „an und für sich weniger bedeutender Bauten in der unmittelbaren Nachbarschaft" zur Geltung komme; Holey 1911, S. 39. 133 Dvořák 1916, S. 1-3. 134 Sonne 2013, S.166-167. 

Kapitel 2 